\title{
Local Absorbent Boundary Condition for Nonlinear Hyperbolic Problems with Unknown Riemann Invariants
}

\author{
Rodrigo R. Paz * , Mario A. Storti and Luciano Garelli \\ Centro Internacional de Métodos Computacionales en Ingeniería (CIMEC), \\ INTEC (CONICET-UNL), Güemes 3450, (S3000GLN) \\ Santa Fe, Argentina
}

\begin{abstract}
A general methodology for developing absorbing boundary conditions for general nonlinear hyperbolic advective-diffusive equations with unknown Riemann invariants is presented. In problems where the Riemann invariants (RI) are known (e.g. the flow in a shallow rectangular channel, the gas flow equations), the imposition of non-reflective boundary conditions is straightforward. In problems where Riemann invariants are unknown (e.g. the flow in a non-rectangular channels, the stratified 2D shallow water flows) it is possible to impose that kind of conditions analyzing the projection of the Jacobians of advective flux functions onto normal directions to fictitious surfaces or boundaries. The advantage of the method is that it is very easy to implement in a finite element code and is only based on computing the advective flux functions (and the their Jacobian projections), then, imposing non-linear constraints via Lagrange Multipliers or Penalty Methods. The application of the dynamic absorbing boundary conditions to typical wave propagation problems with unknown Riemann invariants, like non-linear Saint-Venant system of conservation laws for non-rectangular and non-prismatic 1D channels and stratified 1D/2D shallow water equations, is presented. Also, the new absorbent/dynamic condition can handle automatically the change of Jacobians structure when the flow regime changes from subcritical to supercritical and viceversa, or when recirculating zones are present in regions near fictitious walls.
\end{abstract}

Key words: absorbent boundary condition, Riemann Invariants, hyperbolic PDE's.

\footnotetext{
* Corresponding author.

Email addresses: rodrigop@ intec.unl . edu . ar (Rodrigo R. Paz),

mario.storti@gmail.com (Mario A. Storti), lgarelli@intec.unl.edu.ar (Luciano Garelli).

URLs: http://www. cimec.org.ar/rodrigop, http://www. cimec.org.ar (Rodrigo R. Paz).
} 


\section{Introduction}

Special care must be taken when deciding the number and which boundary conditions have to be imposed at each part of an artificial boundary because in many instances this is a very difficult task and sometimes these conditions are not clear. For hyperbolic problems the decision is based on the number of incoming characteristics $n_{+}$and the quantities known for each problem. On one hand, if the number of conditions imposed on the boundary is in excess they are absorbed through spurious shocks at the boundary. On the other hand, if less conditions are imposed, then the problem is mathematically ill posed and numerical solutions will explode or exhibit spurious oscillations. Even if the number of imposed boundary conditions is correct, this does not guarantee that the boundary conditions are non-reflective. Dealing with models in infinite or large domains implies the introduction of an artificial boundary distant as far as possible from the region of interest. The simplest choice is to impose a boundary condition, assuming that the flow far from the region of interest is undisturbed. However, the boundary condition can be freely chosen so as to give the best solution for a given position of the boundary. Nevertheless, this position is often too far and the computational cost in 2D and 3D problems increases rapidly. Boundary conditions that tend to give the solution as if the domain were infinite are called generally "absorbing" $(\mathrm{ABC})$ or "non reflective" (NRBC). ABC's allows to put the artificial boundary closer to the region of interest for a given admissible error. Of course, the advantage of putting the artificial boundary closer to the region of interest is the reduction in computational cost due to a smaller domain. However, in some cases, like for instance the solution of the Helmholtz equation on exterior domains, using absorbing boundary conditions is required since using a non absorbing boundary condition (like Dirichlet or Neumann) may lead to a lack of convergence of the problem, because these conditions are completely reflective and therefore, wave energy is trapped in the domain, producing false resonance modes.

There are basically two approaches for the design of ABC's, non-local and local. Non-local boundary conditions are usually more accurate than local ones but expensive. In the limit, a non-local $\mathrm{ABC}$ may reproduce the effect of the whole external problem onto the boundary, i.e., even maintaining a fixed position of the artificial boundary the $\mathrm{ABC}$ may give a convergent solution while refining the interior mesh. In general these ABC's are non-local, i.e., its discrete operator is a dense matrix (adding extra computational cost compared to local operators). Non-local boundary conditions exist and are popular for the simpler linear operators, like potential flow problems and frequency domain analysis of wave problems, like the Helmholtz equations for acoustics or the Maxwell equations [5,4,3,7,11,6,2].

The discrete operator for local absorbing boundary conditions is usually sparse but has a lower order accuracy. These kind of ABC's are popular for more complex non-linear fluid dynamic problems, like compressible or incompressible, NavierStokes equations or the inviscid Euler equations. An excellent review has been written by Tsynkov [15]. Nycander et al. [9] have proposed an absorbent bound- 
ary condition for the particular problem of 1D two layers stratified shallow flows based on the barotropic and baroclinic modes and their characteristic variables. The absorbent boundary conditions proposed in this paper are based on the true states of problem variables on each layer and on the analysis of the projection of the Jacobians of advective flux functions onto normal directions to fictitious surfaces as would be explained in next sections.

In order to have an $\mathrm{ABC}$ not any $n_{+}$conditions must be imposed at the boundary but exactly those $n_{+}$corresponding to the incoming characteristics. This can be determined through an eigenvalue $\left(\lambda_{i}\right)$ decomposition problem of the advective flux Jacobian $(\mathbf{A})$ at the boundary (i.e., $\sum_{i}\left(\lambda_{i}(\mathbf{A} \cdot \hat{\mathbf{n}})<0\right)$ ).

In many cases, the number of incoming characteristics may change during the computation. For instance, in Saint-Venant's model it is common that the flow goes from subcritical to supercritical in certain parts of an outlet (fictitious) boundary. In 1D or 2D model this means passing from one imposed boundary condition to none. This is illustrated in the typical problem of a flow passing through a sluice gate (figure 1). The flow upstream of the sluice gate is subcritical. Then, it accelerates to the transcritical and supercritical state as it passes under the gate, which serves as sort of "nozzle". Further downstream the flow shocks back to subcritical regime because the downstream height is too high to maintain supercritical flow. Depending on where the artificial outflow boundary is located (i.e., supercritical or subcritical zone), the number of boundary conditions to be imposed and the structure of the Jacobians associated to this problem will change. When flow of a compressible gas is considered, the fluid state can evolute from subsonic to supersonic and the number of incoming and outgoing characteristics will change too. The change

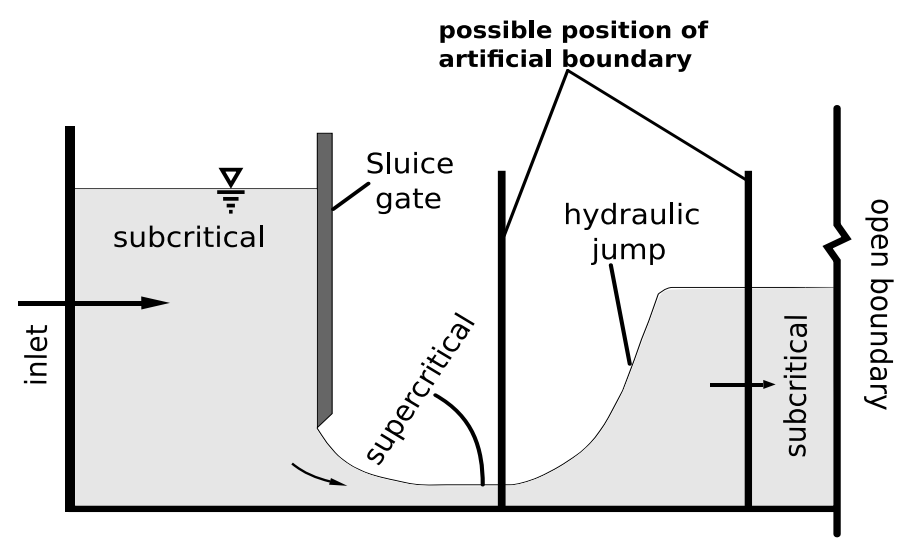

Fig. 1. flow through a sluice gate, flow changes from subcritical to supercritical

of the number of imposed boundary conditions at a given point of the boundary is hard to implement from the computational point of view since it involves the change of the structure of the Jacobian matrix. The solution proposed here is to impose these conditions through Lagrange multipliers or penalization techniques. The main objective of this paper is to discuss numerical aspects related to the use of this techniques and how one can impose absorbing boundary conditions when 
the problem at hand have no Riemann invariants (in a mathematical closed form, see section §3.5) in a mathematical closed form using the scheme presented in [12] for gasflow equations.

The idea of imposing absorbent/dynamics boundary conditions is based on analyzing the projection of the Jacobians of advective flux functions onto normal directions to fictitious surfaces or boundaries. Therefore, working in the characteristic base, the incoming waves can be neglected at fictitious wall obtaining a nonreflective wall. The advantage of the method is that it is very easy to implement in a finite element code and is based on imposing non-linear constraints via Lagrange Multipliers and/or Penalty Methods (see sections $\S 3.7 .1$ and $\S 3.7 .2$ ).

\section{General advective-diffusive systems of equations}

Consider the advective-diffusive system of equations in conservative form

$$
\frac{\partial \mathcal{H}(\mathbf{U})}{\partial t}+\frac{\partial \mathcal{F}_{c, j}(\mathbf{U})}{\partial x_{j}}=\frac{\partial \mathcal{F}_{d, j}(\mathbf{U}, \nabla \mathbf{U})}{\partial x_{j}}+\mathbf{G}
$$

Here $\mathbf{U} \in \mathbb{R}^{n}$ is the state vector, $t$ is time, $\mathcal{F}_{c, j}, \mathcal{F}_{d, j}$ are the advective and diffusive fluxes respectively, $\mathbf{G}$ is a source term including, for instance, gravity acceleration or external heat sources, and $x_{j}$ are the spatial coordinates.

The notation is standard, except for the "generic enthalpy function" $\mathcal{H}(\mathbf{U})$. The enthalpy function allows the inclusion of conservative equations in terms of nonconservative variables. Some well-known hyperbolic advective-diffusive systems of equations studied in this paper may be cast in this general setting as shown in following sections.

\subsection{Shallow water equations}

Shallow water equations describe the open flow of fluids over regions whom characteristic dimensions are much larger than the depth.

$$
\begin{aligned}
\mathbf{U}_{p} & =[h, \mathbf{u}]^{T}, \\
\mathbf{U} & =\mathbf{U}_{c}=[h, h \mathbf{u}]^{T}, \\
\mathcal{H}(\mathbf{U}) & =\mathbf{U}, \\
\mathcal{F}_{c, j} n_{j} & =\left[\begin{array}{c}
h(\mathbf{u} \cdot \hat{\mathbf{n}}) \\
h(\mathbf{u} \cdot \hat{\mathbf{n}}) \mathbf{u}+1 /{ }_{2} g h^{2} \mathbf{I}
\end{array}\right] .
\end{aligned}
$$

where $h$ is the fluid depth, $\mathbf{u}$ the velocity vector, $\mathbf{U}_{p}, \mathbf{U}_{c}$ the primitive and conservative variables, $g$ the gravity acceleration. We assume that the height of the bottom 
with respect to a fixed datum is constant. If this is not so, additional terms must be included in the source term G, but this is irrelevant for the absorbing boundary condition issue. If the channel bed has a variable topography the non-conservative form of shallow water equations must be used.

\subsection{Open channel flow}

Flow in a channel can be cast in advective form as follows

$$
\begin{aligned}
\mathbf{U}_{p} & =[h, u]^{T}, \\
\mathbf{U} & =\mathbf{U}_{c}=[A, Q]^{T}, \\
\mathcal{H}(\mathbf{U}) & =\mathbf{U}, \\
\mathcal{F} & =\left[\begin{array}{c}
Q \\
Q^{2} / A+F
\end{array}\right] .
\end{aligned}
$$

where $h$ and $u$ are water depth and velocity (as in the shallow water equations). $A(h)$ is the section of the channel occupied by water for a given water height $h$. It then defines the geometry of the channel. For instance

- Rectangular channels: $A(h)=w h, w=$ width.

- Triangular channels: $A(h)=2 h^{2} \tan \theta / 2$; with $\theta=$ angle opening.

- Circular channel:

$$
\begin{aligned}
A(h) & =\int_{h^{\prime}=0}^{h} \sqrt{2 R h-h^{2}} \mathrm{~d} h \\
& =\theta R^{2}-w(h)(R-h) / 2
\end{aligned}
$$

where $R$ is the radius of the channel, $w(h)=2 \sqrt{2 R h-h^{2}}$ is the waterline for a given water height and $\theta=\operatorname{atan}[w /(2(R-h))]$ is the angular aperture.

$Q=A u$ is the water flow rate. $F(h)$ is a function defined by

$$
F(h)=\int_{h^{\prime}=0}^{h} A\left(h^{\prime}\right) \mathrm{d} h^{\prime} .
$$

Again, for the sake of simplicity, we restrict to the case of constant channel section an channel depth. For more general situations, other terms can be included in the source and diffusive terms, but they are not needed for the discussion of absorbing boundary conditions. For rectangular channels the equations reduce to those for one dimensional shallow water equations.

Channel flow is very interesting since it is in fact a family of different 1D hyperbolic systems depending on the area function $A(h)$. Riemann invariants are only known for rectangular and triangular channel shapes (see section $\S 3.5$ ). 


\subsection{Stratified shallow water flows}

Another physical model where Riemann invariants have not a mathematical closed form is the flow of a multi-layer fluid in channels.

This kind of physical model exists for instance when flow takes place on a mountainous terrain over plain areas or dense distribution of torrents combined with heavy rainfall. Floods carrying sediments frequently cause collapses, landslides, etc. The numerical model describes multi-layer shallow flows in which the superposed layers differ in density, velocity in a two-dimensional domain.

Consider an $n$-layer model, the layers are indexed by $i$ with per-layer constant density $\rho_{i}$. The thickness of the layer $i$ is $h_{i}(\mathbf{x}, t)$ while the height of the bottom is $h_{0}(\mathbf{x}, t) . i=1$ is the bottom layer, $i=n$ is the top layer. Setting $z_{0}=h_{0}$ then the interfaces between layers are at $z_{i}=z_{i-1}+h_{i}$. The in-plane velocity $u_{i}^{1,2}(\mathbf{x}, t)$ is considered to be constant in each sub-layer and as in one layered shallow water equations, the vertical velocity is averaged and thereafter eliminated. Then the system of mass and momentum conservation equations with $\mathbf{U}=\mathbf{U}_{c}=\left\{h_{i}, h_{i} u_{i}\right\}^{T}$ are:

$$
\begin{aligned}
d_{i} u_{i}+g z_{i x}+g z_{i y} & =-\frac{1}{\rho_{i}} p_{i x}, \\
d_{i} h_{i}+h_{i} u_{i x}+h_{i} u_{i y} & =0 \text { for } i=1,2, . ., n .
\end{aligned}
$$

where

$$
d_{i} f=f_{t}+u_{i} f_{x}
$$

and if the top surface is free

$$
p_{i}=\sum_{j=i+1}^{n} \rho_{j} g\left(z_{j}-z_{j-1}\right)
$$

For the case $n=2$ and traction free at top and bottom surfaces, the stratified shallow water equations are (denoting $u_{i}^{1}=u_{i}$ and $u_{i}^{2}=v_{i}$ ) 


$$
\begin{aligned}
& \frac{\partial h_{1}}{\partial t}+ \frac{\partial}{\partial x}\left(h_{1} u_{1}\right)+\frac{\partial}{\partial y}\left(h_{1} v_{1}\right)=0 \\
& \frac{\partial h_{2}}{\partial t}+\frac{\partial}{\partial x}\left(h_{2} u_{2}\right)+\frac{\partial}{\partial y}\left(h_{2} v_{2}\right)=0 \\
& \frac{\partial}{\partial t}\left(h_{1} u_{1}\right)+\frac{\partial}{\partial x}\left(h_{1}\left(u_{1}\right)^{2}+\frac{1}{2} g h_{1}^{2}\right)+\frac{\partial}{\partial y}\left(h_{1} u_{1} v_{1}\right)+ \\
&+g h_{1} \frac{\partial}{\partial x}\left(z_{0}+\frac{\rho_{2}}{\rho_{1}} h_{2}\right)=0 \\
& \frac{\partial}{\partial t}\left(h_{1} v_{1}\right)+\frac{\partial}{\partial x}\left(h_{1} u_{1} v_{1}\right)+\frac{\partial}{\partial y}\left(h_{1}\left(v_{1}\right)^{2}+\frac{1}{2} g h_{1}^{2}\right)+ \\
&+g h_{1} \frac{\partial}{\partial y}\left(z_{0}+\frac{\rho_{2}}{\rho_{1}} h_{2}\right)=0 \\
& \frac{\partial}{\partial t}\left(h_{2} u_{2}\right)+\frac{\partial}{\partial x}\left(h_{2}\left(u_{2}\right)^{2}+\frac{1}{2} g h_{2}^{2}\right)+\frac{\partial}{\partial y}\left(h_{2} u_{2} v_{2}\right)+g h_{2} \frac{\partial}{\partial x}\left(z_{0}+h_{1}\right)=0 \\
& \frac{\partial}{\partial t}\left(h_{2} v_{2}\right)+\frac{\partial}{\partial x}\left(h_{2} u_{2} v_{2}\right)+\frac{\partial}{\partial y}\left(h_{2}\left(v_{2}\right)^{2}+\frac{1}{2} g h_{2}^{2}\right)+g h_{2} \frac{\partial}{\partial y}\left(z_{0}+h_{1}\right)=0
\end{aligned}
$$

Equations (9) cannot be written in conservation form. The classical hydrostatic pressure assumption is adopted throughout all layers and along the interfaces.

In the general case of $n$ layers and 2D model, the system (6) has $3 n$ waves that propagate inside the domain. In the particular case of the two-layers shallow water equations (eqs. (9)), six waves propagate upstream and downstream at speeds $\lambda_{i}, i$ : $1 . .6$ in both $x$ and $y$ directions.

\subsection{Finite element method (FEM)}

The discretization of physical models described above is made by means of the Finite Element Petrov-Galerkin Method using the SUPG (Streamline Upwind PetrovGalerkin $[14,8]$ ) stabilization and the shock capturing operator [13], which is specially adapted for each flux function of physical models used in this work. The time integration adopted is the trapezoidal rule with $\alpha=1$.

\section{Absorbing boundary conditions}

For steady simulations using time-marching algorithms, it can be shown that the error going towards the steady state propagates like waves, so that absorbing boundary conditions help to eliminate the error from the computational domain. In fact, it can be shown that for strongly advective problems, absorption at the boundaries 
is usually the main mechanism of error reduction (the other mechanism is physical or numerical dissipation in the interior of the computational domain). It has been shown that in such cases the rate of convergence can be directly related to the "transparency" of the boundary condition [1]. In general, absorbing boundary conditions are based on an analysis of the characteristic waves. A key point is to determine which of them are incoming and which are outgoing. Absorbing boundary conditions exist from the simplest first order ones based on a plane wave analysis at a certain smooth portion of the boundary (as will be described below), to the more complex ones that tend to match a full analytic solution of the problem in the external region with that obtained in the internal region. In this paper the usage of absorbing boundary conditions is accomplished in situations where the conditions at the boundary change, so as the number of incoming and outgoing characteristic waves varies during the temporal evolution of the problem, or even when the conditions at the boundary are not well known a priori.

\subsection{Advective diffusive systems in $1 D$}

Let us consider a pure advective system of equations in $1 \mathrm{D}$, i.e., $\mathcal{F}_{d, j} \equiv 0$

$$
\frac{\partial \mathcal{H}(\mathbf{U})}{\partial t}+\frac{\partial \mathcal{F}_{c, x}(\mathbf{U})}{\partial x}=0, \text { in }[0, L] .
$$

If the system is "linear", i.e., $\mathcal{F}_{c, x}(\mathbf{U})=\mathbf{A U}, \mathcal{H}(\mathbf{U})=\mathbf{C U}$ (A and $\mathbf{C}$ do not depend on $\mathbf{U})$, a first order linear system is obtained

$$
\mathbf{C} \frac{\partial \mathbf{U}}{\partial t}+\mathbf{A} \frac{\partial \mathbf{U}}{\partial x}=0
$$

The system is "hyperbolic" if $\mathbf{C}$ is invertible, $\mathbf{C}^{-1} \mathbf{A}$ is diagonalizable with real eigenvalues. If this is the case, it is possible to make the following eigenvalue decomposition for $\mathrm{C}^{-1} \mathrm{~A}$

$$
\mathbf{C}^{-1} \mathbf{A}=\mathbf{S} \boldsymbol{\Lambda} \mathbf{S}^{-1}
$$

where $\mathbf{S}$ is real and invertible and $\Lambda$ is real and diagonal. If a new set of variables is defined $\mathbf{V}=\mathbf{S}^{-1} \mathbf{U}$, then equation (11) becomes

$$
\frac{\partial \mathbf{V}}{\partial t}+\Lambda \frac{\partial \mathbf{V}}{\partial x}=0
$$

Now, the system decouple and each equation is a linear scalar advection equation

$$
\frac{\partial v_{k}}{\partial t}+\lambda_{k} \frac{\partial v_{k}}{\partial x}=0, \quad \text { (no summation over } k \text { ). }
$$

$v_{k}$ are the "characteristic components" and $\lambda_{k}$ are the "characteristic velocities" of propagation. 


\subsection{Linear 1D absorbing boundary conditions}

Assuming that $\lambda_{k} \neq 0$, the absorbing boundary conditions, depending on the sign of $\lambda_{k}$, are

$$
\begin{array}{ll}
\text { if } \lambda_{k}>0: v_{k}(0)=\bar{v}_{k 0} ; & \text { no boundary condition at } x=L \\
\text { if } \lambda_{k}<0: v_{k}(L)=\bar{v}_{k L} ; & \text { no boundary condition at } x=0
\end{array}
$$

This can be put in the follow compact form as

$$
\begin{aligned}
& \boldsymbol{\Pi}_{V}^{+}\left(\mathbf{V}-\overline{\mathbf{V}}_{0}\right)=0 ; \quad \text { at } x=0 \\
& \boldsymbol{\Pi}_{V}^{-}\left(\mathbf{V}-\overline{\mathbf{V}}_{L}\right)=0 ; \quad \text { at } x=L
\end{aligned}
$$

where $\Pi_{V}^{ \pm}$are the projection matrices onto the right/left-going characteristic modes in the $\mathrm{V}$ basis,

$$
\begin{aligned}
& \boldsymbol{\Pi}_{V, j k}^{+}= \begin{cases}1 ; & \text { if } j=k \text { and } \lambda_{k}>0 \\
0 ; & \text { otherwise, }\end{cases} \\
& \boldsymbol{\Pi}^{+}+\boldsymbol{\Pi}^{-}=\mathbf{I} .
\end{aligned}
$$

It can be easily shown that they are effectively projection matrices, i.e., $\Pi^{ \pm} \Pi^{ \pm}=$ $\boldsymbol{\Pi}^{ \pm}$and $\boldsymbol{\Pi}^{+} \boldsymbol{\Pi}^{-}=0$. Coming back to the boundary condition at $x=L$ in the $\mathbf{U}$ basis, it can be written

$$
\Pi_{V}^{-} \mathbf{S}^{-1}\left(\mathbf{U}-\overline{\mathbf{U}}_{L}\right)=0
$$

or, multiplying by $\mathbf{S}$ at the left

$$
\boldsymbol{\Pi}_{U}^{ \pm}\left(\mathbf{U}-\overline{\mathbf{U}}_{0, L}\right)=0, \quad \text { at } x=0, L,
$$

where

$$
\boldsymbol{\Pi}_{U}^{ \pm}=\mathbf{S} \boldsymbol{\Pi}_{V}^{ \pm} \mathbf{S}^{-1},
$$

are the projection matrices in the $\mathbf{U}$ basis. These conditions are completely absorbing for 1D linear advection system of equations (11).

The rank of $\Pi^{+}$is equal to the number $n_{+}$of positive eigenvalues, i.e., the number of right-going waves. Recall that the right-going waves are incoming at the $x=0$ boundary and outgoing at the $x=L$ boundary. Conversely, the rank of $\Pi^{-}$is equal to the number $n_{-}$of negative eigenvalues, i.e., the number of left-going waves (incoming at $x=L$ and outgoing at the $x=0$ boundary).

\subsection{Multidimensional problems}

For multidimensional problems a simplified 1D analysis can be done in the normal direction to the local boundary and with the Jacobian of the advective flux function 
A (equation (12)) replaced with its projection onto the exterior normal $\hat{\mathbf{n}}$, as follows

$$
\begin{aligned}
& \boldsymbol{\Pi}_{n}^{-}(\mathbf{U}-\overline{\mathbf{U}})=0, \\
& \boldsymbol{\Pi}_{n}^{-}=\mathbf{S}_{n} \boldsymbol{\Pi}_{V n}^{-} \mathbf{S}_{n}^{-1}, \\
& \left(\boldsymbol{\Pi}_{V n}^{-}\right)_{j k}= \begin{cases}1 ; & \text { if } j=k \text { and } \lambda_{j}<0 \\
0 ; & \text { otherwise. }\end{cases} \\
& \mathbf{C}^{-1} \mathbf{A}_{n}=\mathbf{S}_{n} \boldsymbol{\Lambda}_{n} \mathbf{S}_{n}^{-1}, \quad\left(\boldsymbol{\Lambda}_{n} \text { diagonal }\right) \\
& \mathbf{A}_{n}=\mathbf{A}_{l} n_{l}
\end{aligned}
$$

These conditions are perfectly absorbing for perturbations reaching the boundary normal to the surface. For perturbations not impinging normally, the condition is partially absorbing, with a reflection coefficient that increases from 0 at normal incidence to 1 for tangential incidence.

\subsection{Absorbing boundary conditions for non-linear problems}

If the problem is non-linear, as the gas dynamics or shallow water equations, then the flux Jacobian $\mathbf{A}$ is a function of the state of the fluid, and then the same happens for the projection matrices $\Pi^{ \pm}$. If it is assumed that the flow is composed of small

perturbations around a state of reference $\mathbf{U}_{\text {ref }}$, then the projection matrix at the state $\mathrm{U}_{\text {ref }}$ can be computed

$$
\Pi\left(\mathbf{U}_{\text {ref }}\right)_{n}^{-}\left(\mathbf{U}-\mathbf{U}_{\text {ref }}\right)=0 \text {. }
$$

However, as long as the fluid state departs from the reference value the condition becomes less absorbing.

\subsection{Riemann invariants based absorbing boundary conditions}

Suppose that for a small interval $t \leq t^{\prime} \leq t+\Delta t$ the state $\mathbf{U}(t)$ is taken as the reference state, then, during this interval $\Pi^{-}(\mathbf{U}(t))$ is taken as the projection operator onto the incoming characteristics and the absorbing boundary conditions are

$$
\Pi^{-}(\mathbf{U}(t))\left(\mathbf{U}\left(t^{\prime}\right)-\mathbf{U}(t)\right)=0
$$

But regarding the equivalent expression (18) it can be written as

$$
\mathbf{l}_{j}(\mathbf{U}) \cdot \mathrm{d} \mathbf{U}=0, \quad \text { if } \lambda_{j}<0
$$

where $\mathbf{l}_{j}$ is the $j$-th left eigenvalue of the normal flux Jacobian. Note that, as $\mathbf{l}_{j}$ is a function of $\mathbf{U}$, this is a differential form on the variable $\mathbf{U}$. If it happens that this is an exact differential, i.e.,

$$
\mu(\mathbf{U}) \mathbf{l}_{j}(\mathbf{U}) \cdot \mathrm{d} \mathbf{U}=\mathrm{d} w_{j}(\mathbf{U}),
$$


for some non-linear function $w_{j}$ and an "integration factor" $\mu(\mathbf{U})$, then it can be imposed

$$
w_{j}(\mathbf{U})=w_{j}\left(\mathbf{U}_{\text {ref }}\right), \quad \text { (for } w_{j} \text { an incoming char.) }
$$

which would be an absorbing boundary condition for the whole non-linear regime. The functions $w_{j}$ are often referred as "Riemann invariants" (RI) for the flux function. The main problem of imposing a condition in this way is that RI are only known for a few set of hyperbolic systems.

For 1D channel flow, Riemann invariants are known for a few channel shapes. For general channel sections they are not known and in addition there is not a general numerical method for computing them. They could be computed by numerical integration of equation (25) along a path in state space, but the integration factor is not known. In addition, a mathematical closed form for the RI of the stratified shallow water model is not known.

For the 2D shallow water equations, the Riemann invariants are well known (see Reference [10]) and

$$
w_{ \pm}=\mathbf{u} \cdot \hat{\mathbf{n}} \pm 2 \sqrt{g h}
$$

and for channel flow they are known only for rectangular and triangular channel shapes. For the triangular case, RI are

$$
w_{ \pm}=\mathbf{u} \cdot \hat{\mathbf{n}} \pm 4 \sqrt{g h} .
$$

For the gas dynamics equations, the well known Riemann invariants are invariant only under isentropic conditions, so that they are not truly invariant. They are

$$
w_{ \pm}=u \pm \frac{2 c}{\gamma-1}
$$

\subsection{Absorbing boundary conditions based on last state}

While integrating the discrete equations in time, the state of the fluid in the previous state can be taken as the reference state

$$
\Pi^{-}\left(\mathbf{U}^{n}\right)\left(\mathbf{U}^{n+1}-\mathbf{U}^{n}\right)=0 .
$$

It is clear that the assumption of linearization is well justified, since in the limit of $\Delta t \rightarrow 0$ it should be $\mathbf{U}^{n+1} \approx \mathbf{U}^{n}$. In fact, (30) is equivalent, for $\Delta t \rightarrow 0$ to (24), so that if Riemann invariants exist, then this scheme preserves them in the limit $\Delta t \rightarrow 0$ and $\Delta x \rightarrow 0$. Hereafter, the proposed strategy is called 'ULSAR' (for Use Last State As Reference).

However, if this scheme is used in the whole boundary, then the flow in the domain is only determined by the initial condition, and it can drift in time due to numerical errors. Also, in a steady state of a certain regime, there is no way to guarantee that the regime will be obtained. For instance, to obtain the steady flow around an aerodynamic profile at a certain Mach number, the initial state with a non perturbed 
constant flow at that condition can be stated, but, it cannot be assured that the final steady flow will preserve that Mach number. In practice, a mix of the strategies are often used, with linear boundary conditions imposed at inlet regions and absorbing boundary conditions based on last state on the outlet regions.

\subsection{Imposing non-linear absorbing boundary conditions}

In this section, the integration of the absorbing boundary conditions in a numerical code is discussed. For linear systems, the discrete version of equation (11) is of the form

$$
\begin{aligned}
& \mathbf{C} \frac{\mathbf{U}_{0}^{n+1}-\mathbf{U}_{0}^{n}}{\Delta t}+\mathbf{A} \frac{\mathbf{U}_{1}^{n+1}-\mathbf{U}_{0}^{n+1}}{h}=0, \\
& \mathbf{C} \frac{\mathbf{U}_{k}^{n+1}-\mathbf{U}_{k}^{n}}{\Delta t}+\mathbf{A} \frac{\mathbf{U}_{k+1}^{n+1}-\mathbf{U}_{k-1}^{n+1}}{2 h}=0, \quad k \geq 1
\end{aligned}
$$

where $\mathbf{U}_{k}^{n}$ is the state at grid point $k$ at time $t^{n}=n \Delta t$. It is assumed a constant mesh step size of $h$, i.e., $x_{k}=k h$, and the boundary located at the mesh node $x_{0}=0$. Several simplifications were assumed here, no source or upwind terms, and a simple discretization based on centered finite differences was used. Alternatively, it can be thought as a pure Galerkin FEM discretization with mass lumping. Also, backward Euler differencing in time is used.

If the projector onto incoming waves $\boldsymbol{\Pi}_{U}^{+}$has rank $n_{+}=n$, then $\boldsymbol{\Pi}_{U}^{+}=\mathbf{I}$ and the absorbing boundary condition reduces to $\mathbf{U}=\mathbf{U}_{\text {ref }}$ (being $\mathbf{U}_{\text {ref }}$ a given value or $\mathbf{U}_{0}^{n}$ for ULSAR). This happens for instance in a supercritical inlet for free surface fluid dynamics or an inlet boundary for linear advection. In this case it is replaced the balance equation for the boundary node (the first equation in (31)) with the absorbing condition $\mathbf{U}=\mathbf{U}_{\text {ref }}$, keeping the balance between equations and unknowns.

Conversely, if the projector onto incoming waves $\Pi_{U}^{+}$has rank $n_{+}=0$, then $\Pi_{U}^{+}=\mathbf{0}$ and the absorbing boundary condition reduces to not imposing anything. This happens for instance in a supercritical outlet in shallow water flows or an outlet boundary for linear advection. In this case the absorbing condition $\mathbf{U}=\mathbf{U}_{\text {ref }}$ is discarded. Again, the number of equations and unknowns is maintained.

The case is more complicated when $0<n_{+}<n$. It cannot be added the absorbing condition (either (19), (26) or (30)), because the boundary balance equation cannot be discarded or maintained.

There are at least two strategies for imposing these non-linear boundary conditions. One possibility is to replace the boundary balance equation for the outgoing waves with a null first derivative condition. Then a discrete version can be generated with finite difference approximations. (This requires, however, a structured mesh at least near the boundary). The other possibility is to resort to the use of Lagrange multipliers or penalization techniques. One advantage of using Lagrange multipliers or penalization is that not only the boundary conditions coefficients can easily be changed for non-linear problems, but also the number of imposed boundary conditions. This is important for problems where the number of incoming characteristics 
can not be easily determined a priori, or for problems where the flow regime is changing from subcritical to supercritical, or the flow reverts. In the rest of this section the second strategy will be described in detail.

In the base of the characteristic variables $\mathrm{V},(31)$ can be written as

$$
\begin{aligned}
& \frac{\mathbf{V}_{0}^{n+1}-\mathbf{V}_{0}^{n}}{\Delta t}+\Lambda \frac{\mathbf{V}_{1}^{n+1}-\mathbf{V}_{0}^{n+1}}{h}=0, \\
& \frac{\mathbf{V}_{k}^{n+1}-\mathbf{V}_{k}^{n}}{\Delta t}+\Lambda \frac{\mathbf{V}_{k+1}^{n+1}-\mathbf{V}_{k-1}^{n+1}}{2 h}=0, \quad k \geq 1
\end{aligned}
$$

For the linear absorbing boundary conditions (19) it should be imposed

$$
\boldsymbol{\Pi}_{V}^{+}\left(\mathbf{V}_{\text {ref }}\right)\left(\mathbf{V}_{0}-\mathbf{V}_{\text {ref }}\right)=0
$$

while discarding the equations corresponding to the incoming waves in the first rows of (32). Here $\mathbf{U}_{\text {ref }} / \mathbf{V}_{\text {ref }}$ is the state about which the linearization is done.

\subsubsection{Using Lagrange multipliers}

This can be done, via Lagrange multipliers in the following way

$$
\begin{aligned}
\boldsymbol{\Pi}_{V}^{+}\left(\mathbf{V}_{\mathrm{ref}}\right)\left(\mathbf{V}_{0}-\mathbf{V}_{\mathrm{ref}}\right)+\boldsymbol{\Pi}_{V}^{-}\left(\mathbf{V}_{\mathrm{ref}}\right) \mathbf{V}_{\mathrm{lm}} & =0, \\
\frac{\mathbf{V}_{0}^{n+1}-\mathbf{V}_{0}^{n}}{\Delta t}+\Lambda \frac{\mathbf{V}_{1}^{n+1}-\mathbf{V}_{0}^{n+1}}{h}+\boldsymbol{\Pi}_{V}^{+}\left(\mathbf{V}_{\mathrm{ref}}\right) \mathbf{V}_{\mathrm{lm}} & =0 \\
\frac{\mathbf{V}_{k}^{n+1}-\mathbf{V}_{k}^{n}}{\Delta t}+\boldsymbol{\Lambda} \frac{\mathbf{V}_{k+1}^{n+1}-\mathbf{V}_{k-1}^{n+1}}{2 h} & =0, \quad k \geq 1,
\end{aligned}
$$

where $\mathbf{V}_{l m}$ are the Lagrange multipliers for the imposition of the new conditions. On one hand, if $j$ is an incoming wave $\left(\lambda_{j} \geq 0\right)$, then the equation is of the form

$$
\begin{aligned}
v_{j 0}-v_{\text {ref } 0} & =0, \\
\frac{v_{j 0}^{n+1}-v_{j 0}^{n}}{\Delta t}+\lambda_{j} \frac{v_{j 1}^{n+1}-v_{j 0}^{n+1}}{h}+v_{j, l m} & =0, \\
\frac{v_{j k}^{n+1}-v_{j k}^{n}}{\Delta t}+\lambda_{j} \frac{v_{j, k+1}^{n+1}-v_{j k}^{n+1}}{2 h} & =0, \quad k \geq 1 .
\end{aligned}
$$

Note that, due to the $v_{j, 1 m}$ Lagrange multiplier, it can be solved for the $v_{j k}$ values from the first and last rows, while the value of the multiplier $v_{j, l m}$ "adjusts" itself in order to satisfy the equations in the second row.

On the other hand, for the outgoing waves $\left(\lambda_{j}<0\right)$, the equations are

$$
\begin{aligned}
v_{j, l m} & =0, \\
\frac{v_{j 0}^{n+1}-v_{j 0}^{n}}{\Delta t}+\lambda_{j} \frac{v_{j 1}^{n+1}-v_{j 0}^{n+1}}{h} & =0, \\
\frac{v_{j k}^{n+1}-v_{j k}^{n}}{\Delta t}+\lambda_{j} \frac{v_{j, k+1}^{n+1}-v_{j k}^{n+1}}{2 h} & =0, \quad k \geq 1 .
\end{aligned}
$$


So that the solution coincides with the unmodified original FEM equation, and the Lagrange multiplier is $v_{j, l m}=0$.

Coming back to the $\mathrm{U}$ basis, it can be written

$$
\begin{aligned}
\boldsymbol{\Pi}_{U}^{+}\left(\mathbf{U}_{\mathrm{ref}}\right)\left(\mathbf{U}_{0}-\mathbf{U}_{\mathrm{ref}}\right)+\boldsymbol{\Pi}_{U}^{-}\left(\mathbf{U}_{\mathrm{ref}}\right) \mathbf{U}_{\mathrm{lm}} & =0, \\
\mathbf{C} \frac{\mathbf{U}_{0}^{n+1}-\mathbf{U}_{0}^{n}}{\Delta t}+\mathbf{A} \frac{\mathbf{U}_{1}^{n+1}-\mathbf{U}_{0}^{n+1}}{h}+\mathbf{C} \boldsymbol{\Pi}_{U}^{+}\left(\mathbf{U}_{\mathrm{ref}}\right) \mathbf{U}_{\mathrm{lm}} & =0, \\
\mathbf{C} \frac{\mathbf{U}_{k}^{n+1}-\mathbf{U}_{k}^{n}}{\Delta t}+\mathbf{A} \frac{\mathbf{U}_{k+1}^{n+1}-\mathbf{U}_{k-1}^{n+1}}{2 h} & =0, \quad k \geq 1 .
\end{aligned}
$$

\subsubsection{Using penalization}

The corresponding formulas for penalization can be obtained by adding a diagonal term scaled by a small enough regularization parameter $\epsilon$ to the first equation in (37)

$$
\begin{array}{r}
-\epsilon \mathbf{U}_{1 m}+\Pi_{U}^{+}\left(\mathbf{U}_{0}-\mathbf{U}_{\text {ref }}\right)+\Pi_{U}^{-} \mathbf{U}_{1 m}=0, \\
\mathbf{C} \frac{\mathbf{U}_{0}^{n+1}-\mathbf{U}_{0}^{n}}{\Delta t}+\mathbf{A} \frac{\mathbf{U}_{1}^{n+1}-\mathbf{U}_{0}^{n+1}}{h}+\Pi_{U}^{+} \mathbf{U}_{\mathrm{lm}}=0 ;
\end{array}
$$

where, for the moment, the dependence of the projectors on $\mathbf{U}_{\text {ref }}$ is dropped. Eliminating $\mathbf{U}_{\mathrm{lm}}$ from the first and second rows it is obtained

$$
\mathbf{C} \frac{\mathbf{U}_{0}^{n+1}-\mathbf{U}_{0}^{n}}{\Delta t}+\mathbf{A} \frac{\mathbf{U}_{1}^{n+1}-\mathbf{U}_{0}^{n+1}}{h}+\boldsymbol{\Pi}_{U}^{+}\left(\boldsymbol{\Pi}_{U}^{-}+\epsilon \mathbf{I}\right)^{-1} \boldsymbol{\Pi}_{U}^{+}\left(\mathbf{U}_{0}-\mathbf{U}_{\mathrm{ref}}\right)=0 .
$$

Now, using projection algebra it can be shown that

$$
\left(\mathbf{\Pi}_{U}^{-}+\epsilon \mathbf{I}\right)^{-1}=\left(\frac{1}{\epsilon} \boldsymbol{\Pi}_{U}^{+}+\frac{1}{1+\epsilon} \mathbf{\Pi}_{U}^{-}\right)
$$

so that the last term in (39) reduces to $\boldsymbol{\Pi}_{U}^{+}\left(\mathbf{U}_{0}-\mathbf{U}_{\text {ref }}\right)$ and the whole equation is

$$
\mathbf{C} \frac{\mathbf{U}_{0}^{n+1}-\mathbf{U}_{0}^{n}}{\Delta t}+\mathbf{A} \frac{\mathbf{U}_{1}^{n+1}-\mathbf{U}_{0}^{n+1}}{h}+\frac{1}{\epsilon} \mathbf{C} \Pi_{U}^{+}\left(\mathbf{U}_{0}-\mathbf{U}_{\text {ref }}\right)=0 .
$$

Here $1 / \epsilon$ can be taken as a large penalization factor.

\section{Numerical experiments}

Several numerical and theoretical aspects of absorbent boundary conditions are studied in this section. Most of them arising in typical problems in hydrology and hydraulic areas where hyperbolic PDE's govern their behavior. The experiments range from 1D Saint-Venant models for different channel shapes, to 1D and 2D stratified shallow flows and problems with regime change and back-flow at outlet fictitious boundaries. 


\subsection{D shallow water equations}

As explained in precedent sections, RI for 1D Saint-Venant equations are known only for rectangular and triangular channel shapes. So, classical ABC could be used in order to avoid wave reflections at fictitious walls. The next section is devoted to evaluate the performance of ULSAR ABC's proposed here in problems where channel shapes differ from those with known Riemann invariants. Also, the performance of ULSAR conditions is compared with those ABC's based on Riemann characteristics in the full nonlinear range in the case of the shallow-water flow in a rectangular channel. It is shown that both (ULSAR and RI based B.C.'s) local boundary conditions have similar "transparency" properties.

\subsubsection{Circular cross section channel}

Consider a 1D Saint-Venant flow in a very long horizontal and circular section channel with radius $R_{\text {chann }}=1 \mathrm{~m}$. All variables and parameters are dimensionless by selecting $R_{\text {chann }}$ and $g=1 \mathrm{~m} / \mathrm{s}^{2}$ as reference values for length and time scales. Fictitious walls are introduced at $x=0$ and $x=L_{x}=20$. The fluid state is initialized having a strong perturbation in the free surface elevation $h$ which is function of the coordinate $x$ and describing a Gaussian curve triggering full nonlinear effects. The perturbation is

$$
h(x, t=0)=h_{0}+\frac{A}{\sqrt{2 \pi}} \exp ^{-0.5\left(\frac{x-\mu}{\sigma}\right)^{2}}
$$

with $h_{0}=1, A=2, \mu=L_{x} / 2$ and $\sigma=0.04 L_{x}$. The initial velocity $u$ is constant in the whole domain at $t=0$, i.e, $u(x, t=0)=0.5$. For $t>0$ the initial perturbation breaks and two resultant waves (that are not symmetrical due to the initial imposed velocity) move toward the fictitious walls. The non-dimensional number that governs this kind of flow is the Froude number $\left(F_{\mathrm{r}}=|u| / \sqrt{g h}\right)$ which is subcritical in this case. The evolution of the perturbation is simulated using $N_{x}=400$ equalspaced finite elements $\left(\Delta_{x}=L_{x} / N_{x}=0.05\right)$ with SUPG stabilization and trapezoidal temporal scheme with $\Delta t=0.05$. Absorbing boundary conditions based on the ULSAR strategy are applied at both ends $x=0, L$. The set of non-absorbent classical Dirichlet B.C. are $u(x=0, t)=0.5$ and $h\left(x=L_{x}, t\right)=1$.

In Figure (2) the time evolution of the free surface elevation is shown for both type of boundary conditions. Note that the transparency of ULSAR B.C.'s is very high. Impinging waves are completely absorbed as they pass through walls despite the full non-linear character of the flow. Similar results are obtained with other channel sections like, trapezoidal, parabolic or more general sections derived from the basic ones. 
ULSAR B.C. for circular channel section

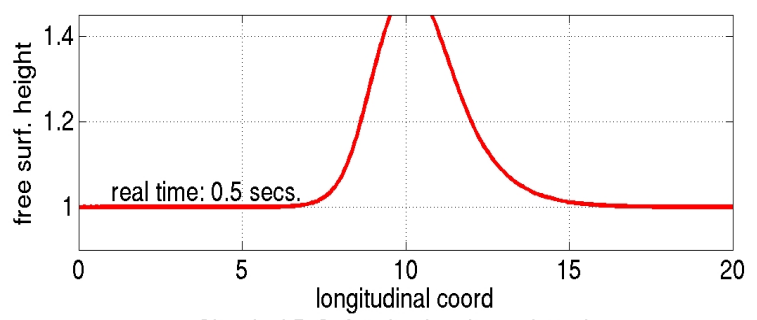

Classical B.C. for circular channel section

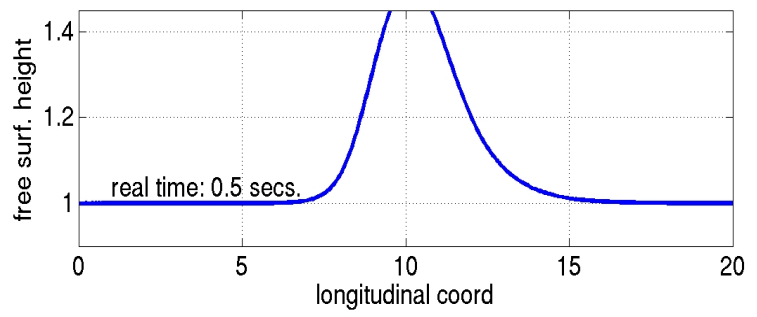

ULSAR B.C. for circular channel section

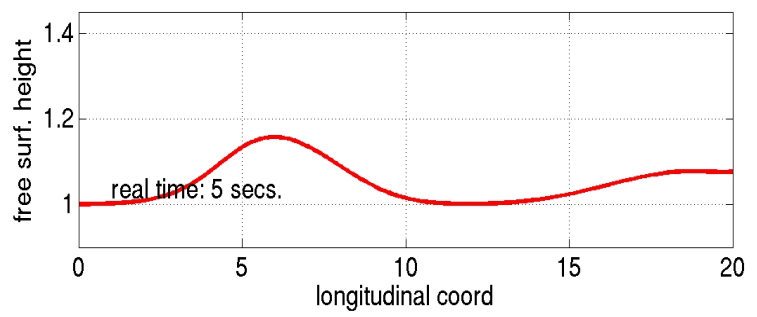

Classical B.C. for circular channel section

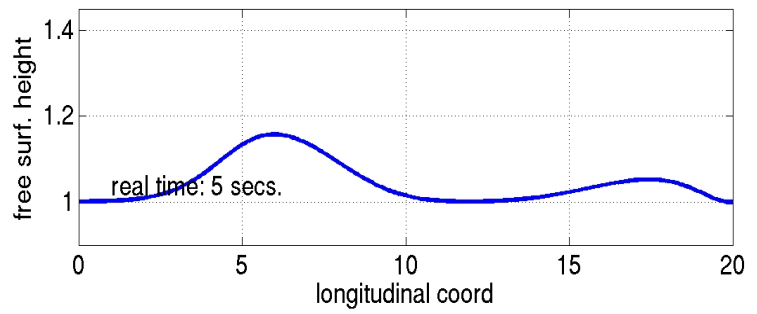

ULSAR B.C. for circular channel section

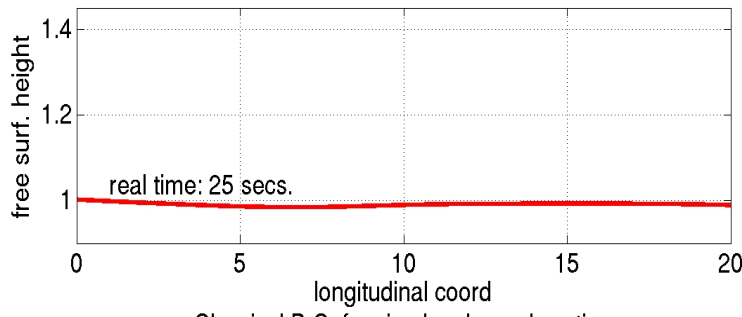

Classical B.C. for circular channel section

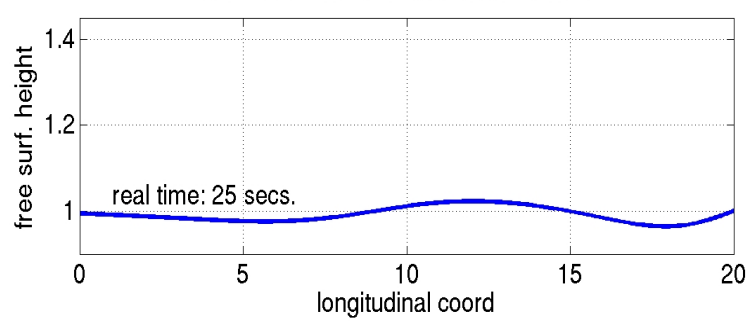

ULSAR B.C. for circular channel section

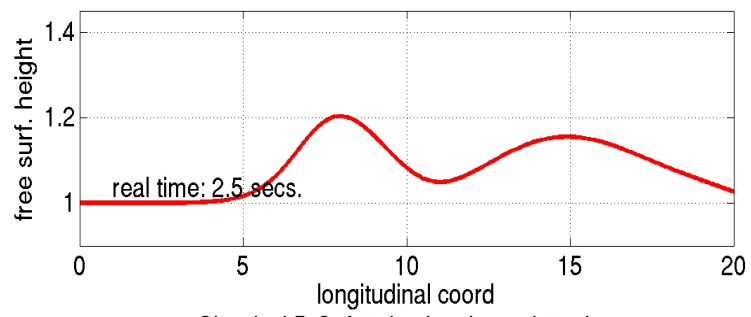

Classical B.C. for circular channel section

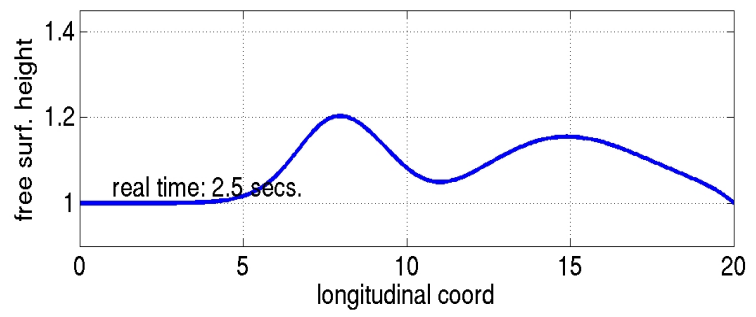

ULSAR B.C. for circular channel section

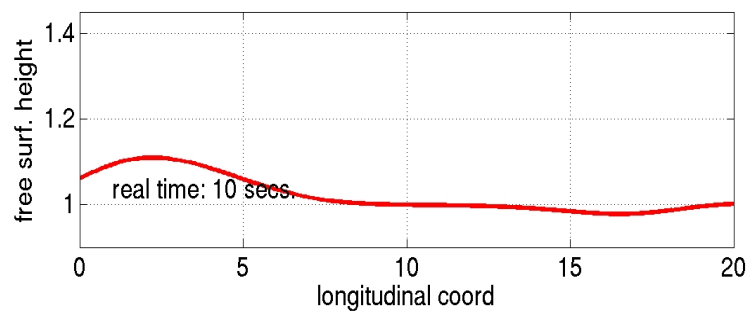

Classical B.C. for circular channel section

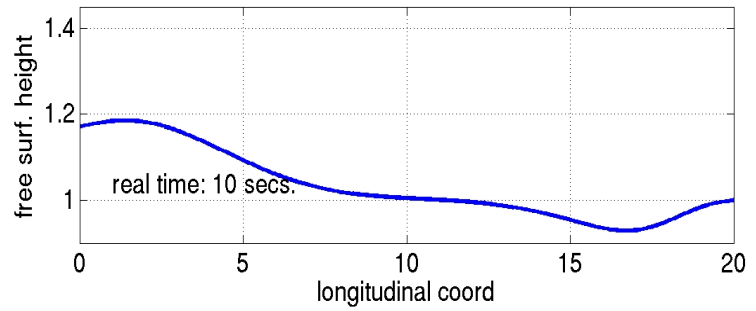

ULSAR B.C. for circular channel section

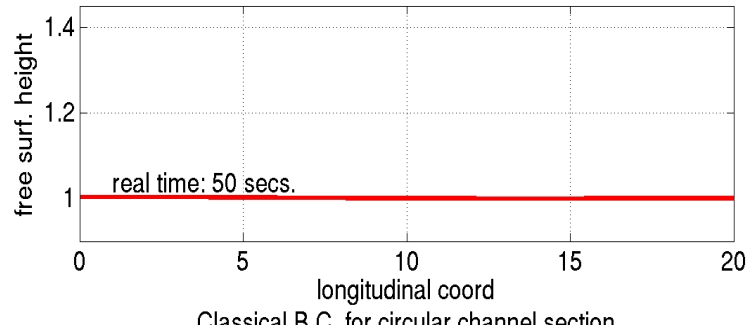

Classical B.C. for circular channel section

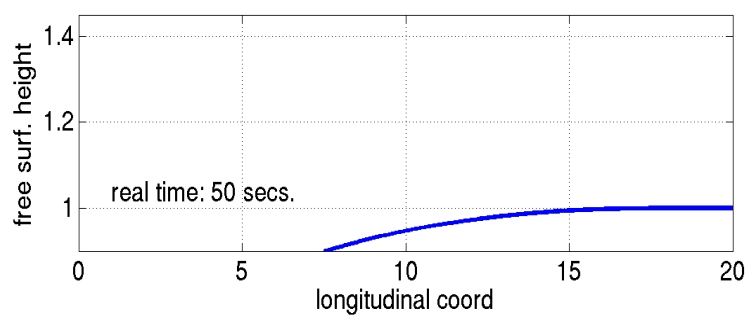

16

Fig. 2. Classical and ULSAR B.C.'s comparison for 1D-SW flow in a circular section channel. 


\subsubsection{Generic shape channel}

This test is similar to the previous one except that the channel section shape is constructed using the most basic shapes or generated by a polygonal curve, for instance. Then, consider the 1D Saint-Venant flow in a channel with cross section as shown in Figure (3). Same parameters, initial and boundary conditions adopted

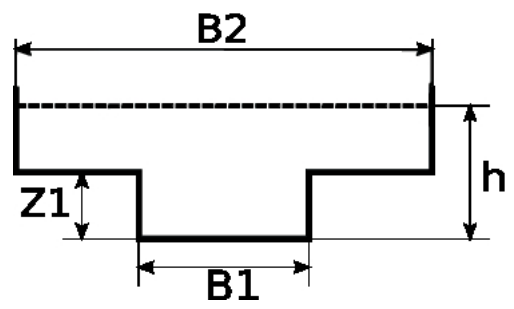

Fig. 3. Cross section definitions

for the circular channel case are used here. Geometrical dimensions are: B1 = $4 \mathrm{~m}, \mathrm{~B} 2=6 \mathrm{~m}$ and $\mathrm{Z} 1=2 \mathrm{~m}$. All variables and parameters are dimensionless by selecting $B 1$ and $g=1 \mathrm{~m} / \mathrm{s}^{2}$ as reference values for length and time scales. Figure (4) show the time evolution of the free surface height using ULSAR B.C.'s and Classical Dirichlet ones. Here, the impinging waves are completely absorbed at walls in the same fashion as in previous example.

\subsection{Rectangular cross section channel: comparison between ULSAR and Rie- mann invariants based $A B C$}

The key point of this test is to show experimentally that the ULSAR conditions are equivalent to the Riemann invariants based conditions. For this purpose, an 1D Saint-Venant flow in a rectangular channel of $1 \mathrm{~m}$ width is considered. Physical and numerical parameters, and the initial and boundary conditions are the same as in previous cases, testing the both conditions in the linear and non-linear ranges.

All variables and parameters are dimensionless by selecting $B=1$ (the channel width) and $g=1 \mathrm{~m} / \mathrm{s}^{2}$.

Figure (5) show the behavior of the free surface and the transparency of both approaches. In Figures (6) and (7) the differences, in both variables $u(x=0, t)$ and $h(x=0, t)$ as the simulation proceed are shown. It is clear from figures that differences are several orders of magnitude less than the values of variables. But perhaps a better way to evaluate the performance of ULSAR B.C.'s, compared to Riemann invariants based conditions, is computing the norm $L_{2}$ of the spatial derivative of $u$ and $h$ as a function of time, i.e., $\|d u / d x\|_{2}$ and $\|d h / d x\|_{2}$. Clearly, this is a measure of the error between the actual fluid state to the steady non-perturbed solution once the waves leave the domain. The error is shown in Figures (8) and (9) putting in evidence that ULSAR conditions are equivalent to RI based one including the linear and non-linear ranges. 
ULSAR B.C. for generic channel section

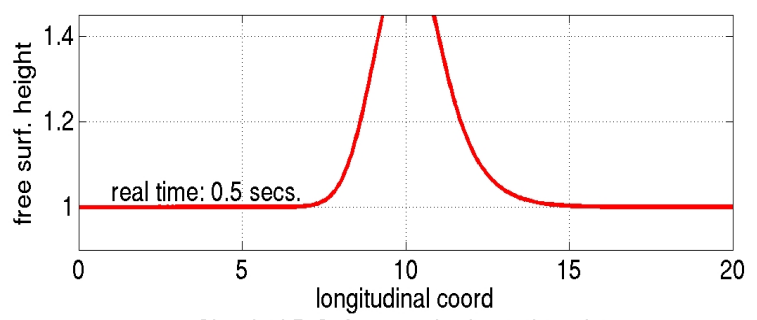

Classical B.C. for generic channel section

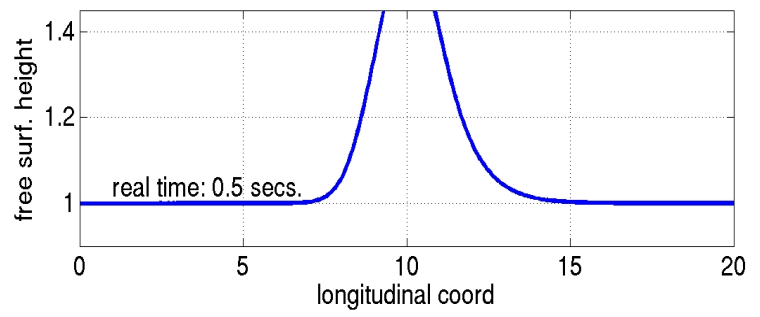

ULSAR B.C. for generic channel section

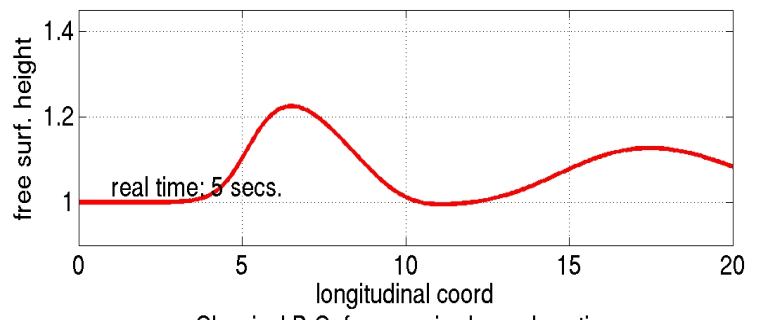

Classical B.C. for generic channel section

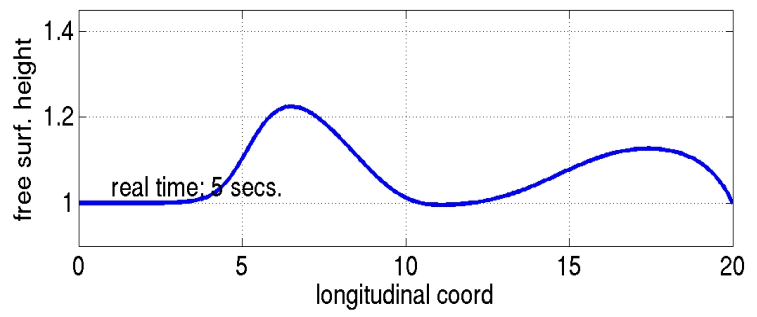

ULSAR B.C. for generic channel section

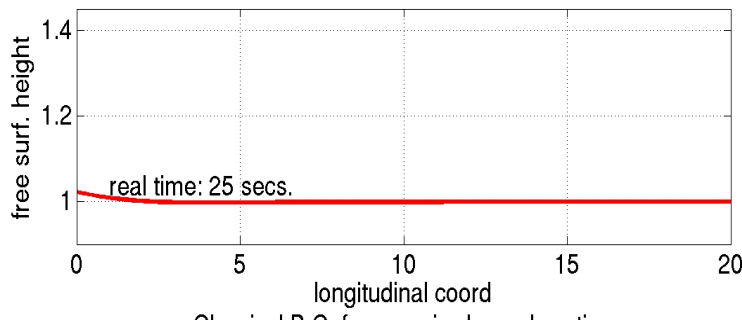

Classical B.C. for generic channel section

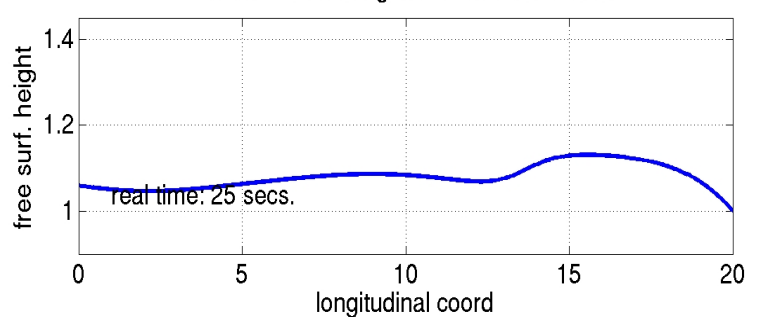

ULSAR B.C. for generic channel section

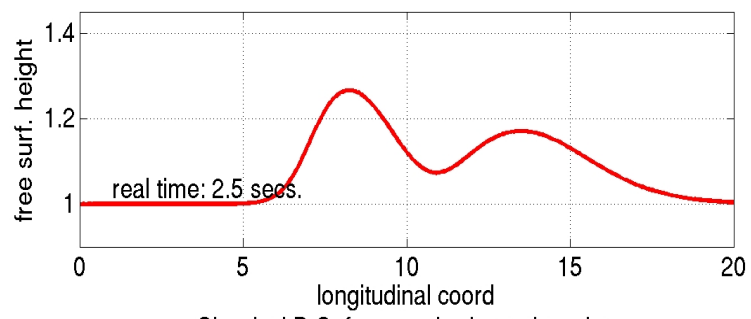

Classical B.C. for generic channel section

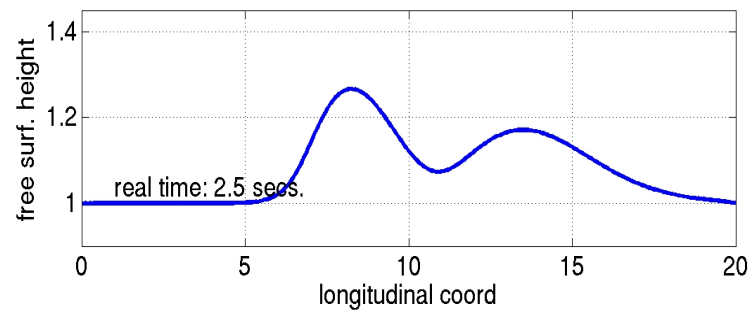

ULSAR B.C. for generic channel section

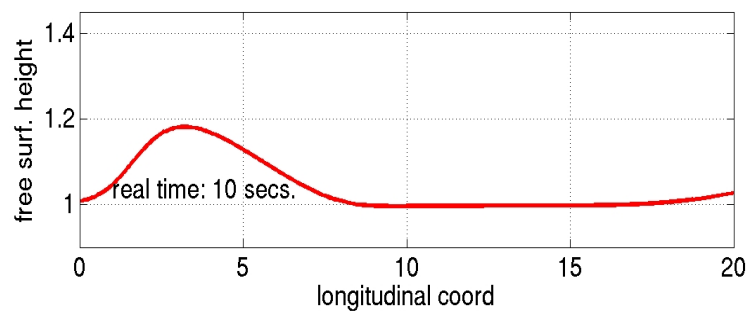

Classical B.C. for generic channel section

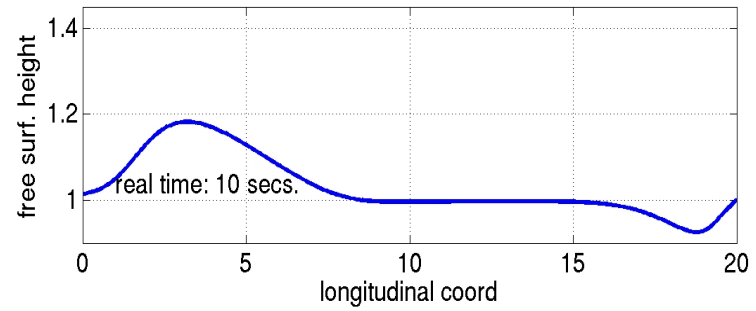

ULSAR B.C. for generic channel section

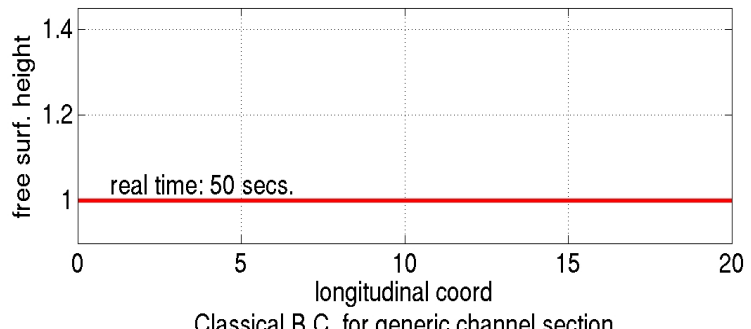

Classical B.C. for generic channel section

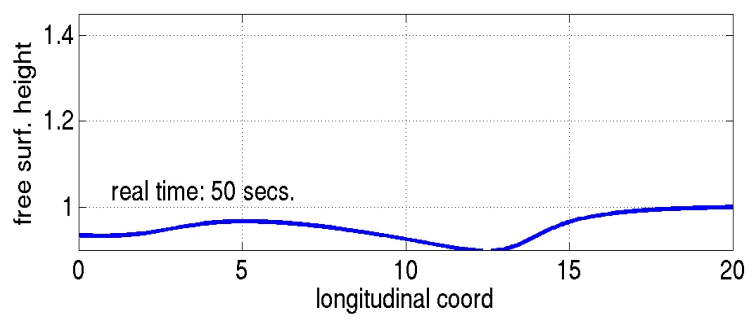

18

Fig. 4. Classical and ULSAR B.C.'s comparison for 1D-SW flow in a channel with a polygonal cross section. 
ULSAR B.C. for rectang. channel section

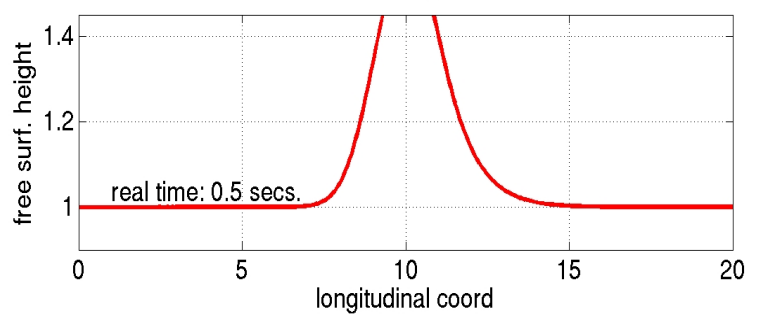

Riemann Invs, based B.C. for rectang. channel section

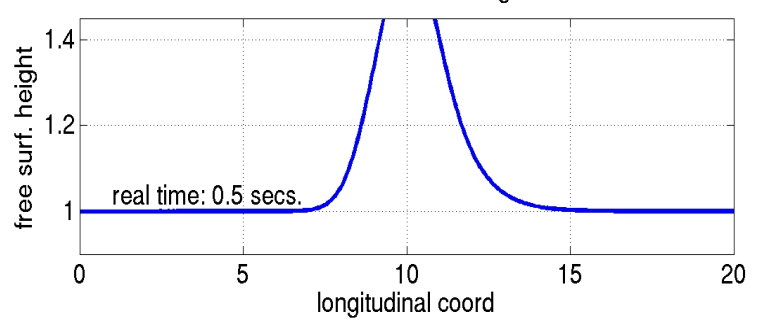

ULSAR B.C. for rectang. channel section

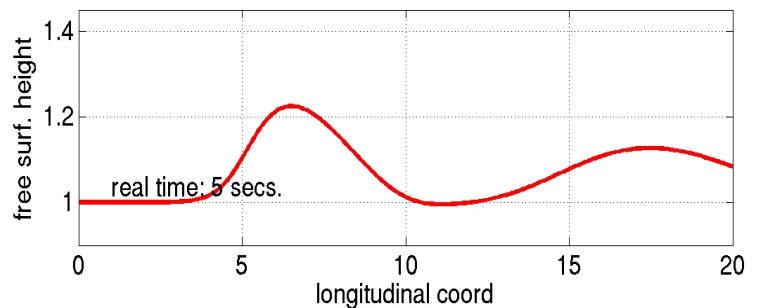

Riemann Invs. based B.C. for rectang. channel section

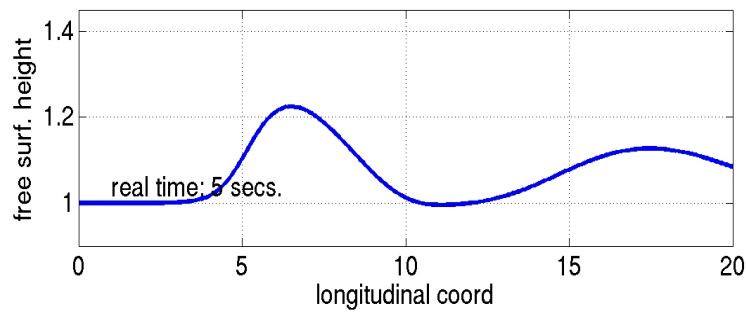

ULSAR B.C. for rectang. channel section

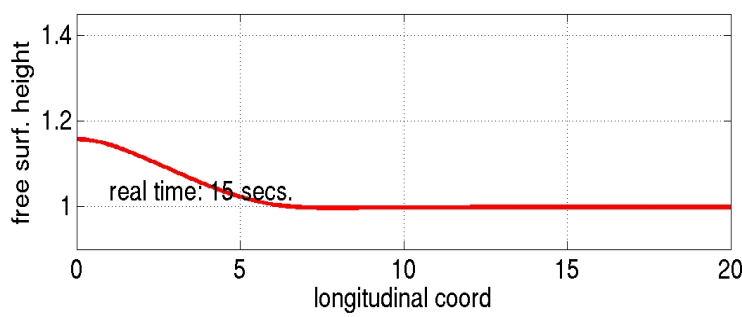

Riemann Invs, based B.C. for rectang. channel section

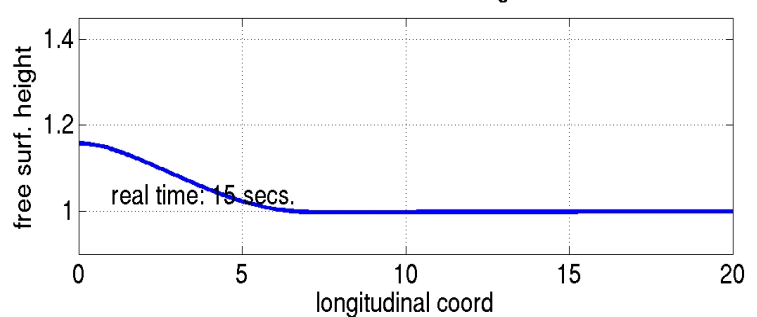

ULSAR B.C. for rectang. channel section

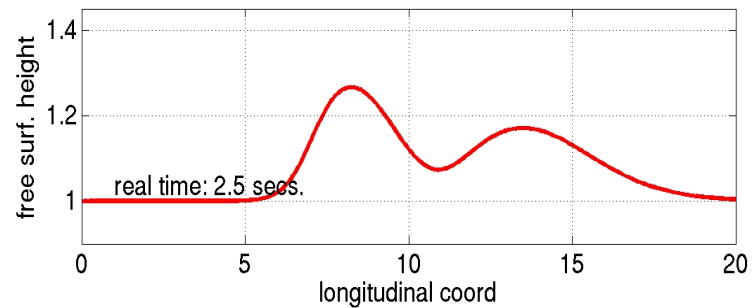

Riemann Invs. based B.C. for rectang. channel section

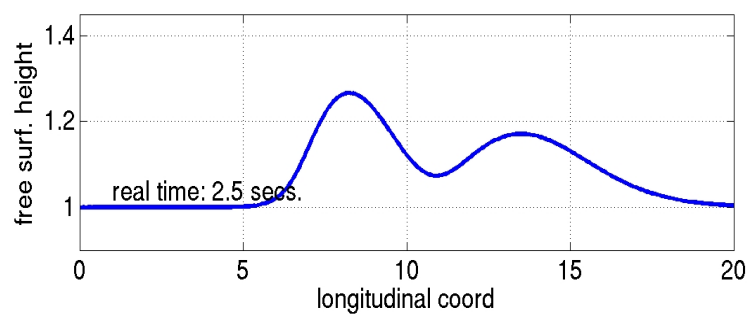

ULSAR B.C. for rectang. channel section

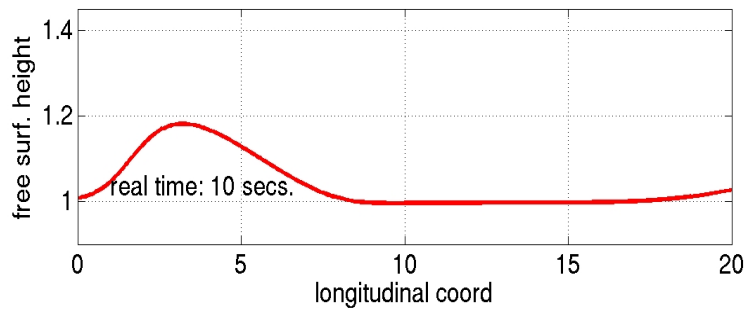

Riemann Invs. based B.C. for rectang. channel section

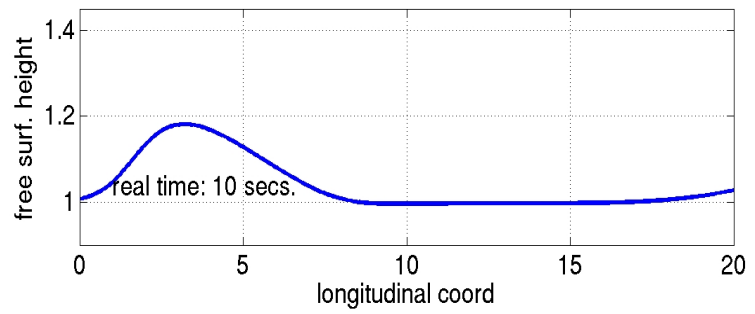

ULSAR B.C. for rectang. channel section

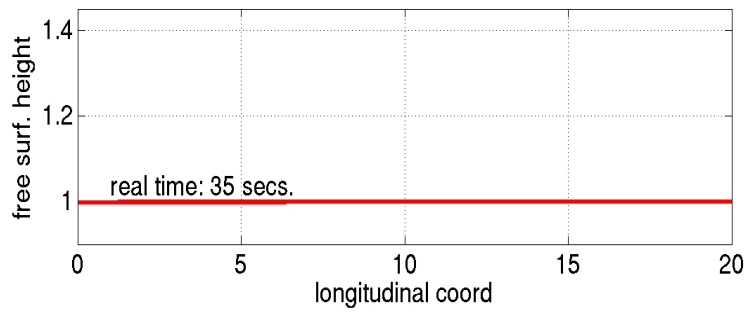

Riemann Invs. based B.C. for rectang. channel section

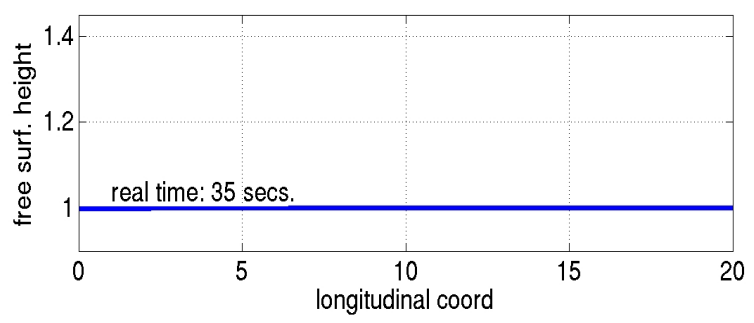

19

Fig. 5. ULSAR and Riemann invariants based ABC's comparison for 1D shallow water flow in a rectangular channel. 


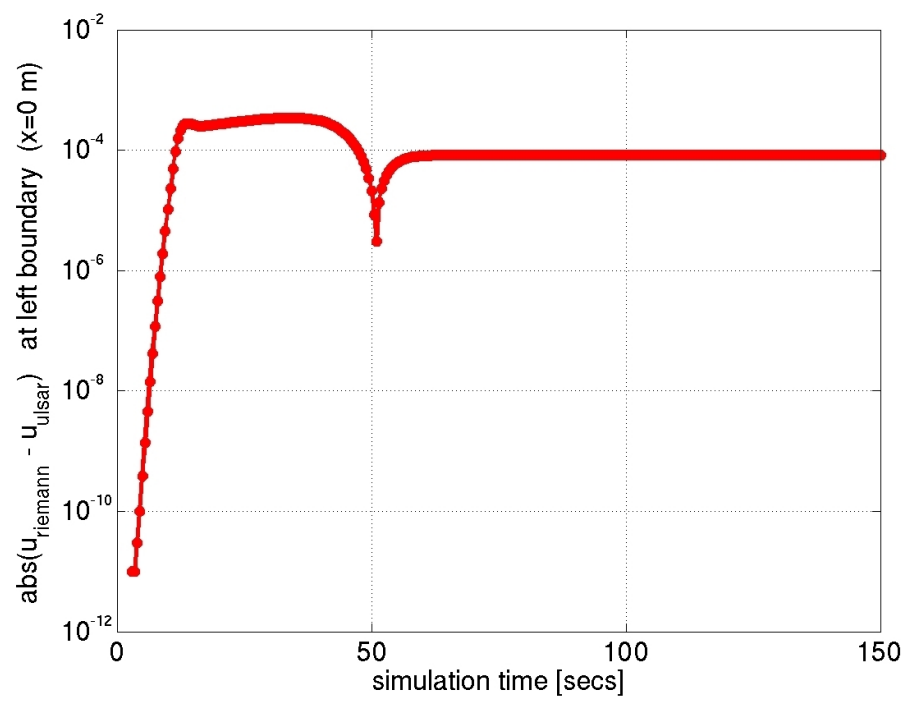

Fig. 6. Differences (velocity) between ULSAR and Riemann invariants based ABC's at left fictitious boundary: the evolution in time for 1D shallow water flow in a rectangular channel.

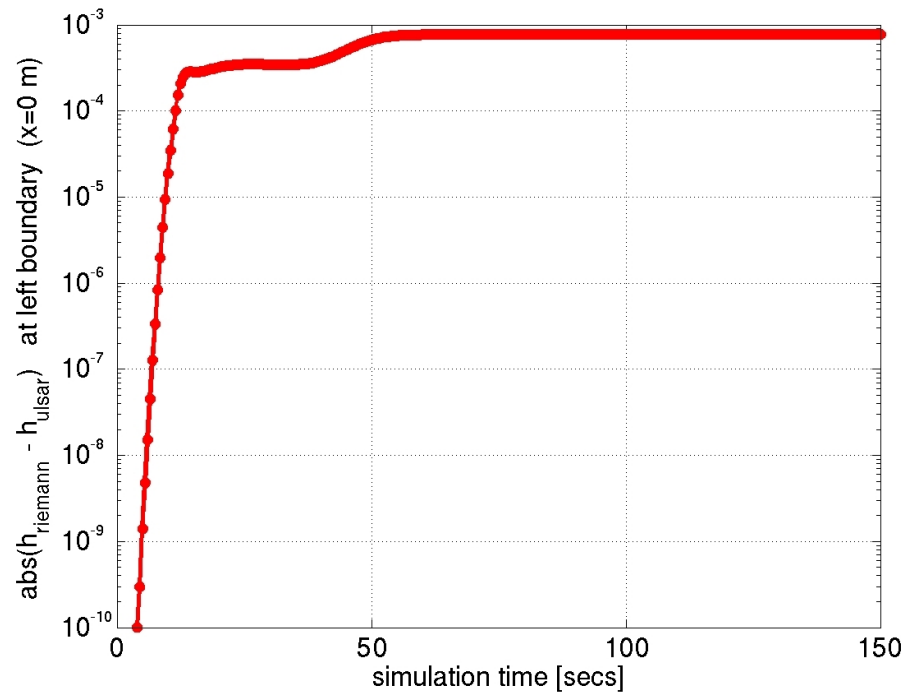

Fig. 7. Differences (free surf. height) between ULSAR and Riemann invariants based $A B C$ 's at left fictitious boundary: the evolution in time for 1D shallow water flow in a rectangular channel.

\subsection{D stratified shallow water equations}

Let us focus this test on the one-dimensional version of equations (6) and (9). This case is similar to the one-layer examples presented above but considering the flow of two immiscible layers over a rectangular unbounded channel. All variables and parameters are dimensionless by selecting $B=1 \mathrm{~m}$ (the channel width) and 


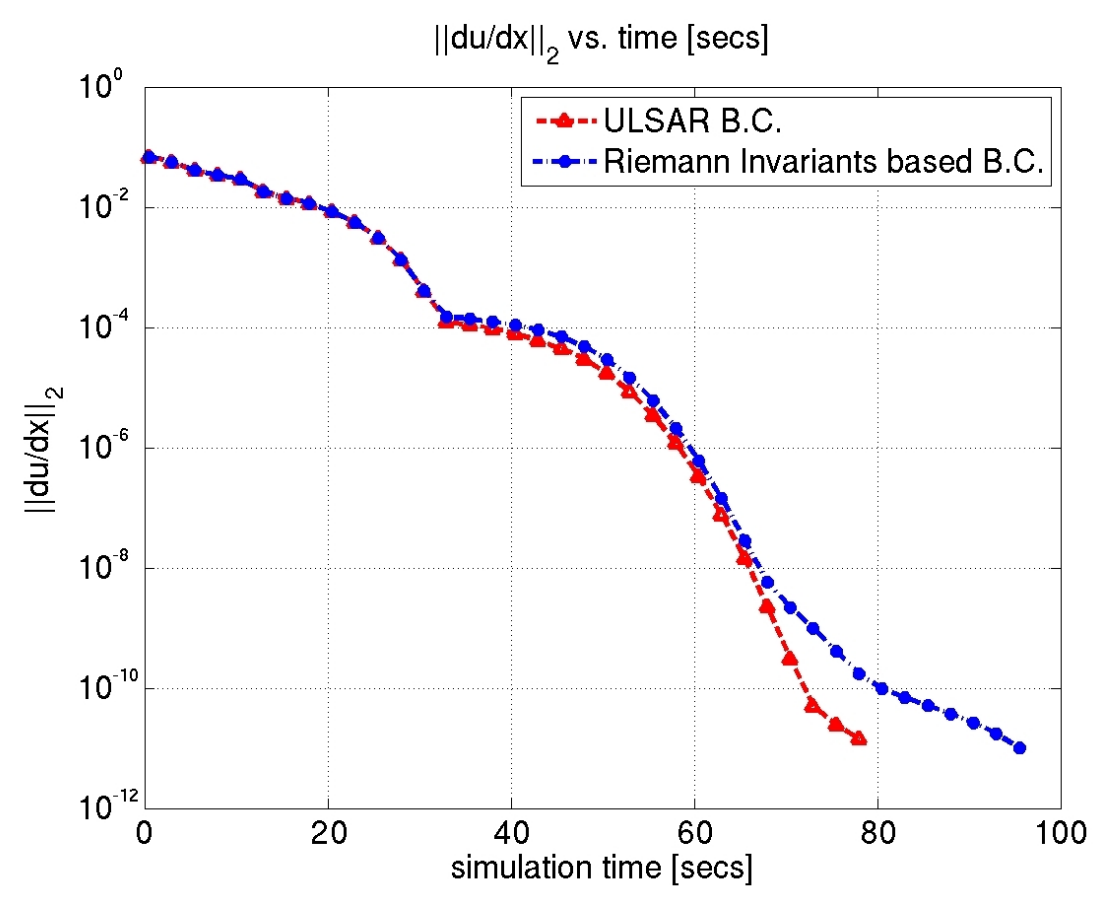

Fig. 8. $\|d u / d x\|_{2}$ vs. time for ULSAR and Riemann invariants based ABC's.

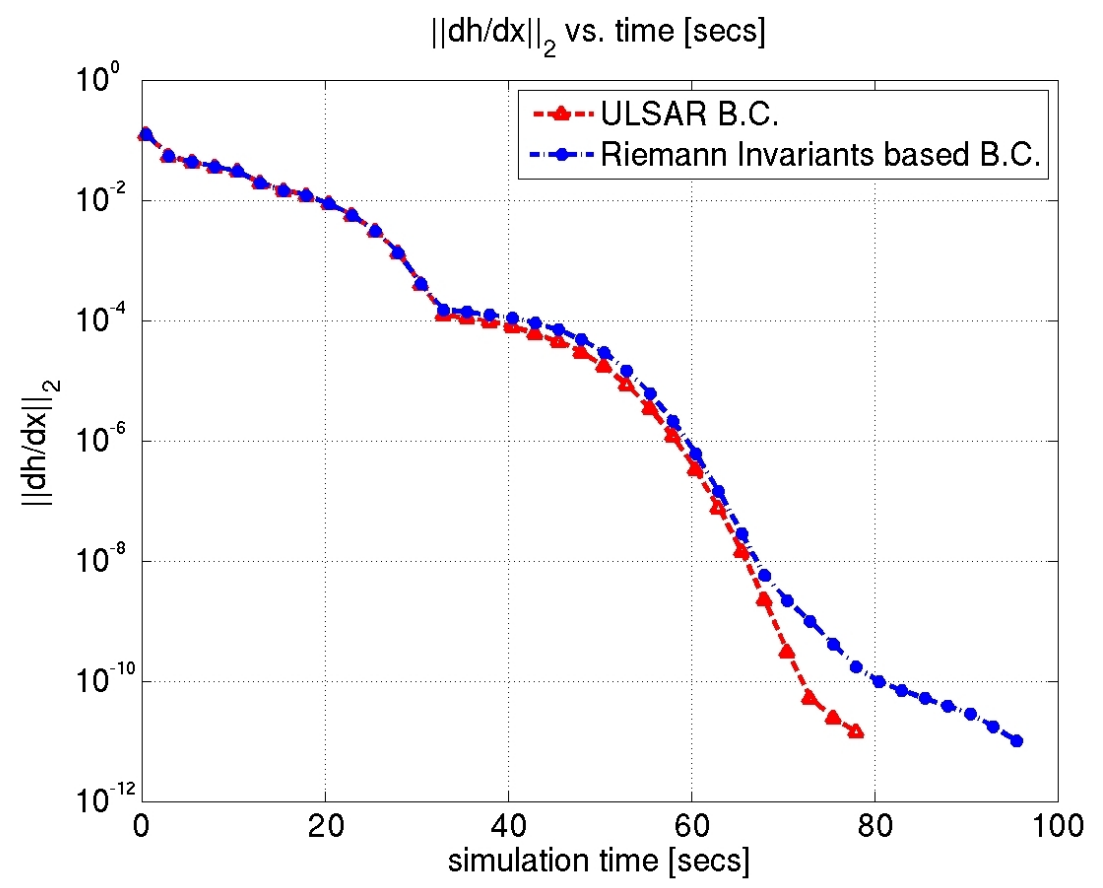

Fig. 9. $\|d h / d x\|_{2}$ vs. time for ULSAR and Riemann invariants based ABC's.

$g=1 \mathrm{~m} / \mathrm{s}^{2}$ as reference values for length and time scales. Absorbing walls are introduced at $x=0$ and $x=L_{x}=20$. The state is initialized with a strong perturbation in the variable $h(x, t=0)$ of the top layer (i.e., $\left.h_{2}(x, t=0)\right)$ describing a centered curve like equation (4.1.1) with $h_{0}=0.5, A=2.5, \mu=L_{x} / 2$ and $\sigma=0.04 L_{x}$. The bottom layer is initialized with a constant height along the 
channel such that $h_{1}=1$. Both layers have an initially low velocity of ( $u_{1}(x, t=$ $\left.0)=u_{2}(x, t=0)=0.15\right)$. Then, the initial perturbation (in the $h$ variable) is decomposed in two non-symmetrical waves travelling in opposite directions and interacting immediately with the bottom layer as shown in Figure (10). There are four characteristic waves in this 1D case. Based on the non-perturbed state two characteristic waves are right-going and two are left-going. The solution with classical B.C. are also plotted in the sequence of Figure (10). $\rho_{1} / \rho_{2}=2$ is used. As shown in figure, high transparency properties of ULSAR ABC's are achieved too for this problem.

\subsection{D stratified shallow water equations: Dam-break problem}

The $2 \mathrm{D}$ version of the two-layer shallow water equations is used to simulate the dam-break phenomenon. All variables and parameters are dimensionless by selecting $h_{1}(x, t=0)=1 \mathrm{~m}$ and $g=1 \mathrm{~m} / \mathrm{s}^{2}$. The computational region is a $20 \mathrm{~m}$ by $20 \mathrm{~m}\left(L_{x}=L_{y}=20\right)$ squared dam channel as shown in Figure 11 with two walls located at the middle of the channel. The separation gap of the walls is 10 . The main channel is horizontal. A mesh of triangles is used and the average element size is 0.2 . The initial condition is such that in both layers the fluid is at rest, so, $u_{1}(x, y, t=0)=u_{2}(x, y, t=0)=v_{1}(x, y, t=0)=v_{2}(x, y, t=0)=0$ and a strong discontinuity in both layers thickness located at the levee station is introduced, i.e., $h_{1}\left(x, y>L_{y} / 2, t=0\right)=h_{2}\left(x, y>L_{y} / 2, t=0\right)=1$, and $h_{1}\left(x, y \leq L_{y} / 2, t=0\right)=h_{2}\left(x, y \leq L_{y} / 2, t=0\right)=0.75$. The density ratio is $\rho_{1} / \rho_{2}=4$ and $g=1$. Friction terms are neglected in this problem and this assumption has no effect when evaluating the transparency of the artificial walls. The time step adopted is $\Delta t=0.025 \mathrm{~s}$. ULSAR absorbent conditions are used in all walls excepting the levee walls where a non-slip condition is imposed (in both layers). Regarding the geometry of the domain and the expansion waves generated at levee, the quality of ULSAR conditions is evaluated for impinging waves that are not only normals to walls but also to waves inciding with angles that can vary from 0 to 90 degrees. As stated in section $\S 2.3$ there are six characteristic waves. It is verified that during the whole simulation, at the outlet (left) wall four characteristic waves are outgoing and two are incoming. The sequence in Figure (11) illustrates the computational results for each layer thickness and the efficiency of proposed conditions. As shown in figure, ULSAR conditions behave quite good as fictitious boundaries including the effect of the angle of incidence of arriving waves.

\subsection{Absorbent B.C. as dynamic B.C: problems with regime change}

The modeling of the free surface fluid flow problem with dynamically regime change is a challenge from several points of view. One of these points is the im- 
ULSAR B.C. for 1D stratified flow

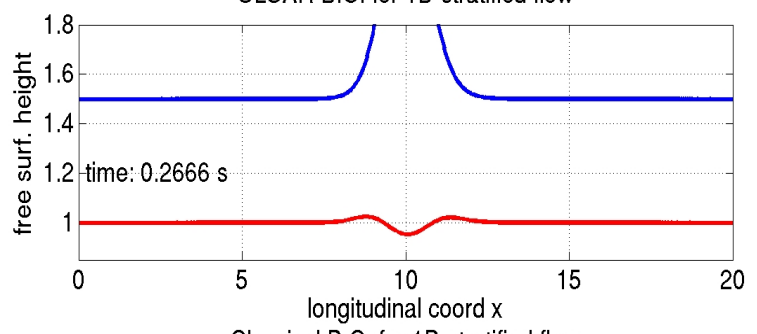

Classical B.C. for 1D stratified flow

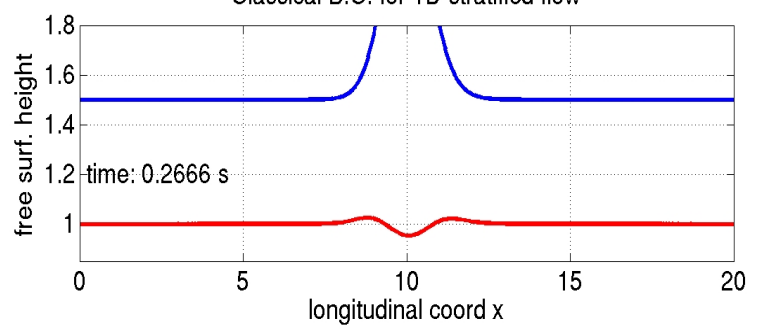

ULSAR B.C. for 1D stratified flow

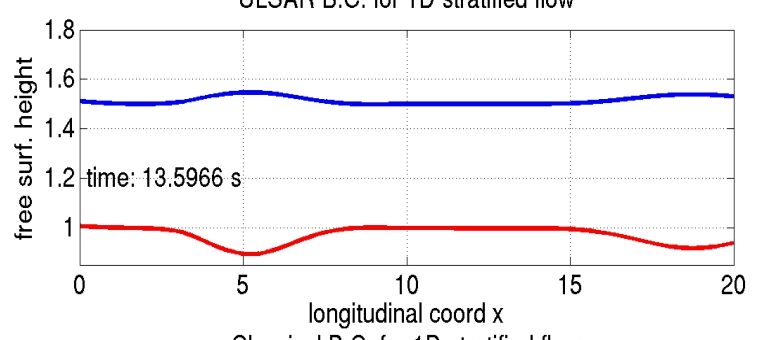

Classical B.C. for $1 D$ stratified flow

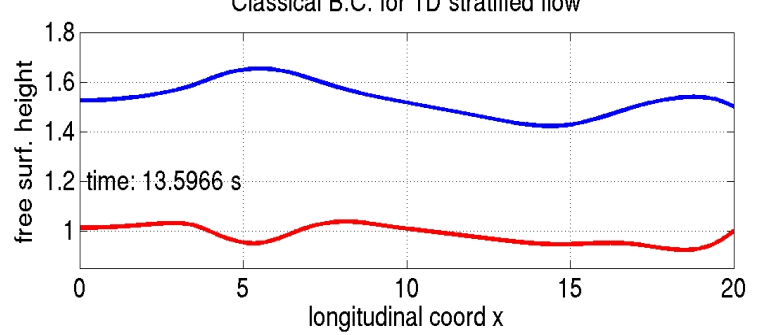

ULSAR B.C. for $1 D$ stratified flow

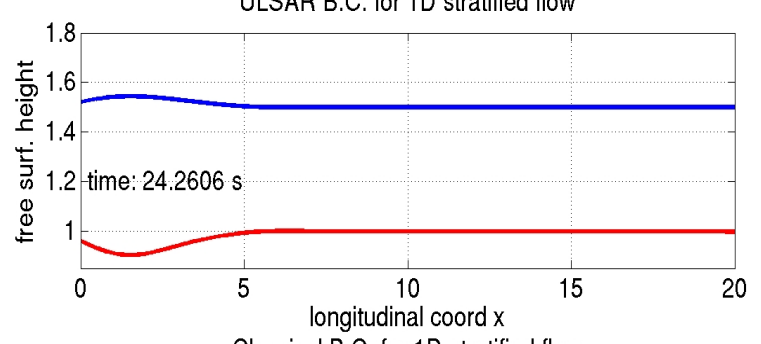

Classical B.C. for $1 D$ stratified flow

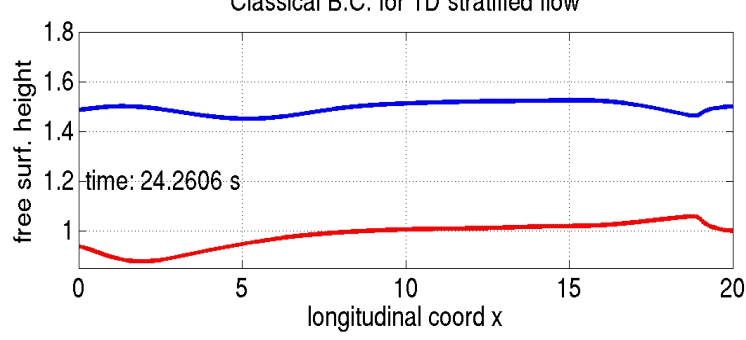

ULSAR B.C. for $1 \mathrm{D}$ stratified flow

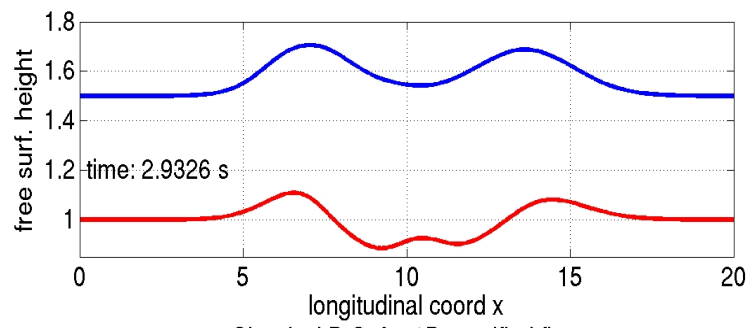

Classical B.C. for 1D stratified flow

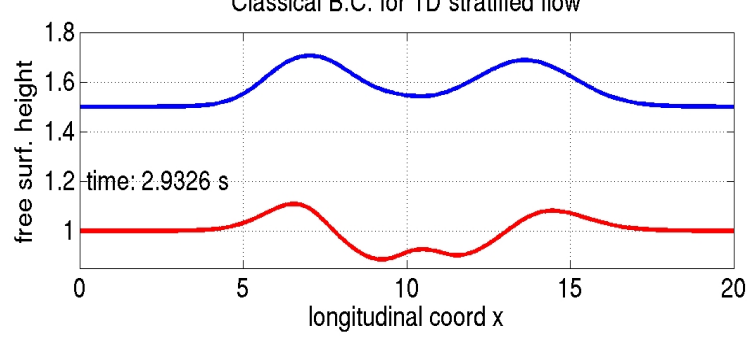

ULSAR B.C. for 1D stratified flow

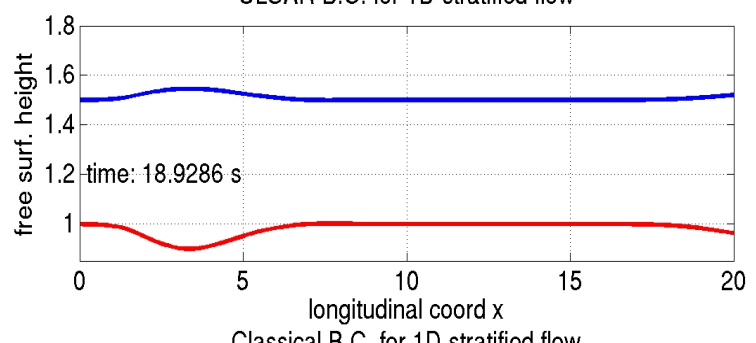

Classical B.C. for 1D stratified flow

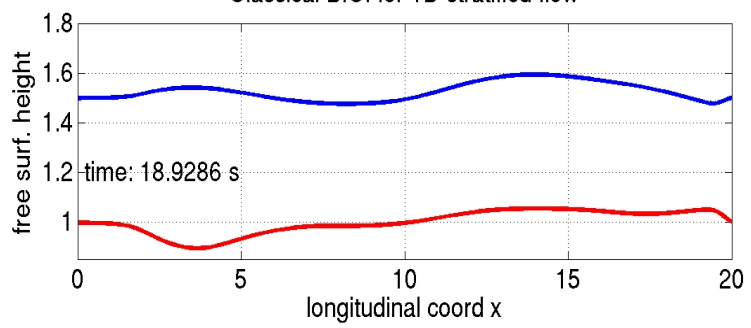

ULSAR B.C. for $1 D$ stratified flow

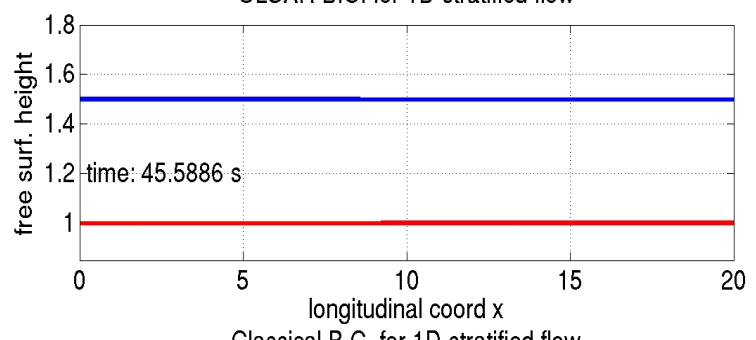

Classical B.C. for 1D stratified flow

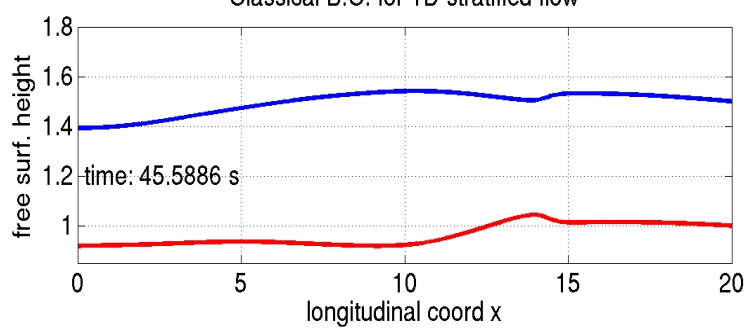

Fig. 10. Classical and ULSAR B.C.'s comparison for 1D-stratified shallow water flow in a rectangular channel. red: bottom layer, blue: top layer. 

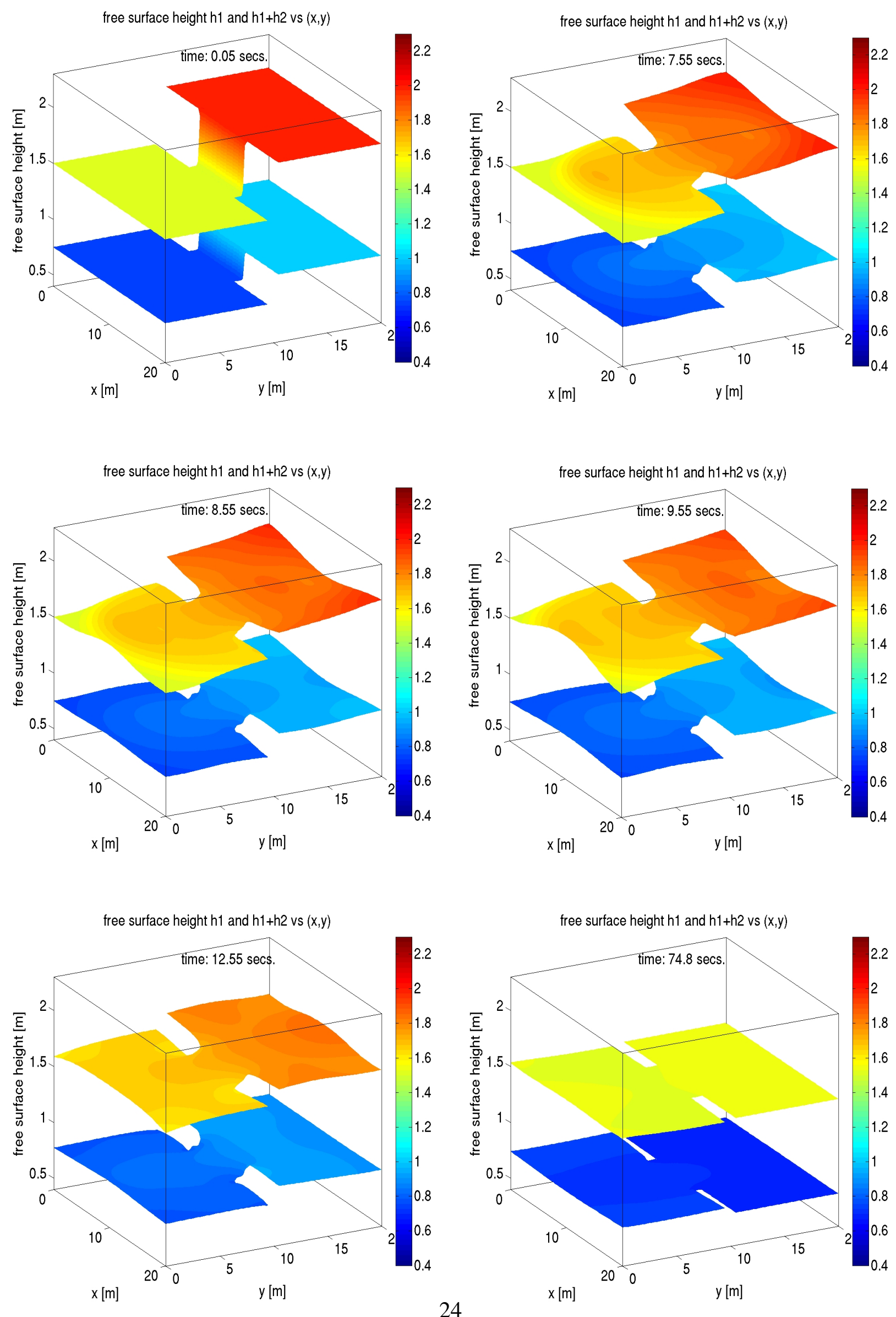

Fig. 11. 2-layer dam break with ULSAR B.C. 
position of boundary conditions that at an outlet wall must be non-reflecting when the unbounded domain is truncated (to save computational resources for instance). Moreover, in such case, the needed conditions at the outlet wall would change from subcritical to supercritical ones (and viceversa) as a shock wave (or a strong discontinuity or a hydraulic jump) appears and propagates toward the boundary. So, the condition must be capable of handling the dynamical change of the Jacobians matrix profile. During the flow computation inside the channel the number of incoming/outgoing characteristics, and therefore the number of Dirichlet conditions to be imposed, will change. Having a boundary condition that can automatically adapt itself to this change is essentially useful in such a problem. In addition, the computational domain is limited to a zone of interest and therefore the CPU time and used memory are drastically reduced. As explained above, imposing absorbent/dynamics boundary conditions is based on analyzing the projection of the Jacobians of advective flux functions onto normal directions to fictitious surfaces. So, when working in the characteristic base, the incoming waves are fixed to zero $\left(v_{k}=0\right)$ at fictitious walls obtaining a non-reflective wall. As shown in section $\S 3.7 .1$, the method has an extra advantage that is very easy to implement based on imposing non-linear constraints.

Let us consider the 2D "subcritical to supercritical" shallow water flow on a channel ( $L_{x}=40 \mathrm{~m}$ long, $L_{y}=20 \mathrm{~m}$ width) with variable bed topography. All variables and parameters are dimensionless by selecting $h(x, y, t=0)=1 \mathrm{~m}$ and $g=1 \mathrm{~m} / \mathrm{s}^{2}$ as reference values for length and time scales. The perturbation introduced on the bed is a parabolic bump located at $(x, y)=(10,10)$, with height $t_{\text {bump }}=0.5$ and diameter $D_{\text {bump }}=12$. At lateral wall $(y=0$ and $y=20) \mathrm{a}$ slip condition is used. Initial state, shown in Figure (12), is obtained from a steady solution of a simulation at low subcritical conditions $((u, v, h)=(0.5,0,1)$ at left inlet wall and ULSAR B.C.'s at right outlet wall). Also, at time $t=0$ the state at the left boundary wall is raised from steady conditions to a supercritical state with $(u, v, h)=(2.4,0,3.1)$. Consequently, a strong hydraulic jump is generated at left wall which travels downstream with velocity $V_{\text {jump }}=(3,0)$. The discontinuity interacts with the bump and is finally absorbed at right boundary leaving a steady supercritical flow in the whole domain. So, the fluid flow evolves from subcritical to supercritical changing automatically and dynamically the flow conditions upstream and downstream as explained in previous chapters. In the sequence of Figure (13) it is shown how ULSAR conditions adapt automatically to give a well-posed dynamic and absorbent condition at artificial walls. The steady supercritical water level (as seen from a fixed reference) is shown in Figure (14).

\subsection{Absorbent B.C. as dynamic B.C: $2 D$ shallow water equations with back-flow at boundaries}

Finally, another interesting aspect of the absorbent/dynamics conditions based on the last state is pointed out in this test where a back-flow bucket is originated at 


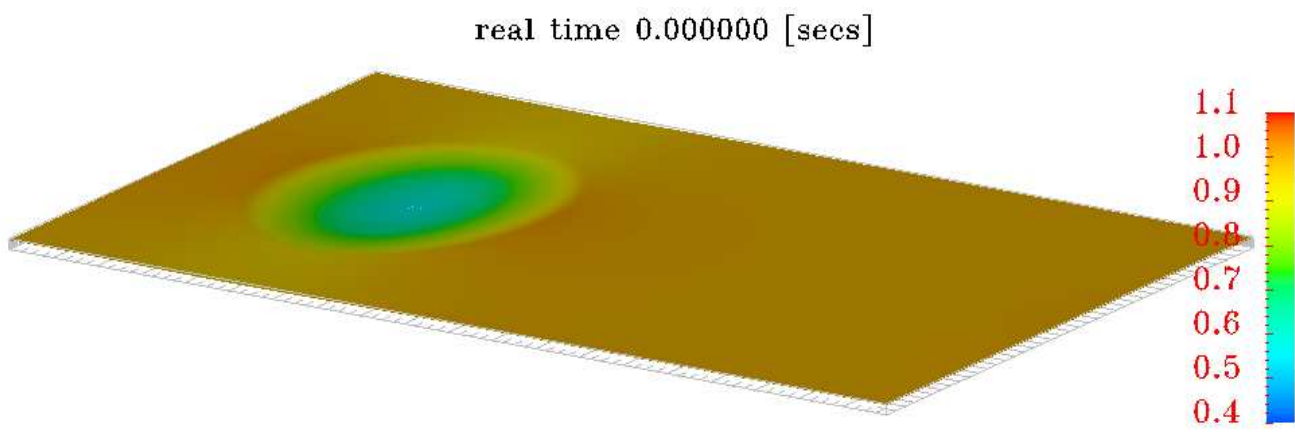

Step $=0--$ color $=$ free surf. elevation

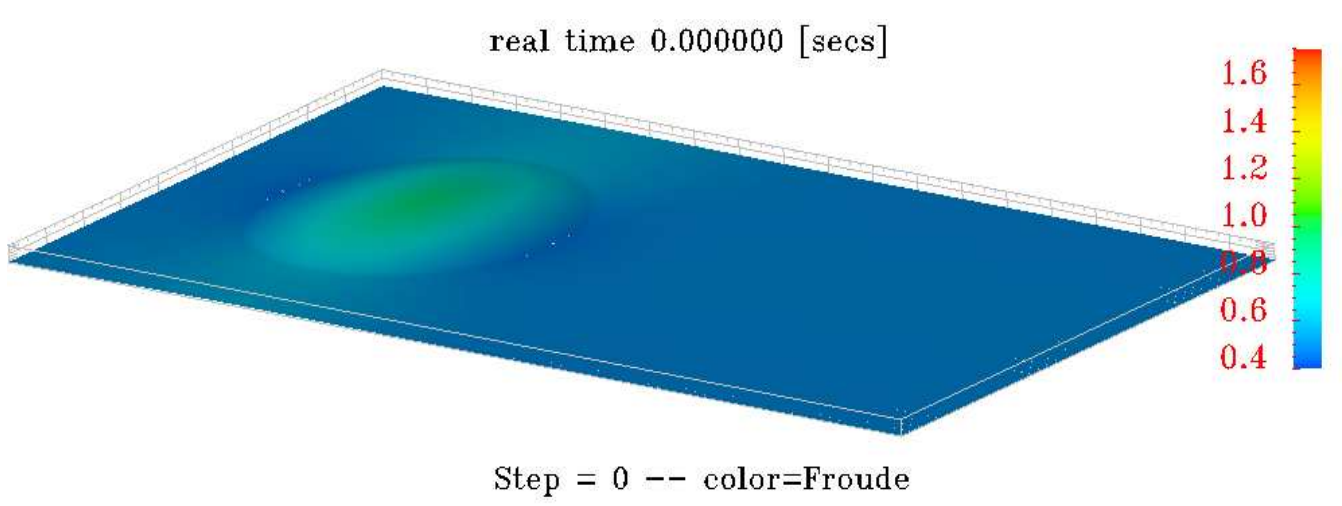

Fig. 12. Initial State: free surface and Froude initial conditions.

an outlet boundary. Regarding that the number and the set of Dirichlet conditions to be imposed depend on that the wall is an outlet or an inlet, and that the domain may have a complicated geometry (e.g., levees, derivation wall, sluice gates, etc.), it could happen that meanwhile the correct set of B.C. at an outlet has been imposed, some emitted vortices can reach the outlet wall and change it to an inlet wall. If classical conditions for the outlet are maintained, the problem could become ill-posed and the solution misbehaving. Figures (15) and (16) show how ULSAR B.C's tackle the problem imposing automatically the correct set of B.C.'s when two vortices, generated at levee walls in the dam break problem, move toward the outlet walls giving recirculation zones at outflow boundary. Figure (15) shows the time evolution of the water surface when the dam brakes and the system reaching a steady state avoiding spurious solutions. Figure (16) shows in detail a corner of the downstream portion of the dam and the fluid entering to the domain (see the velocity vectors) due to the emission of vortices and their contact with the absorbent walls. 

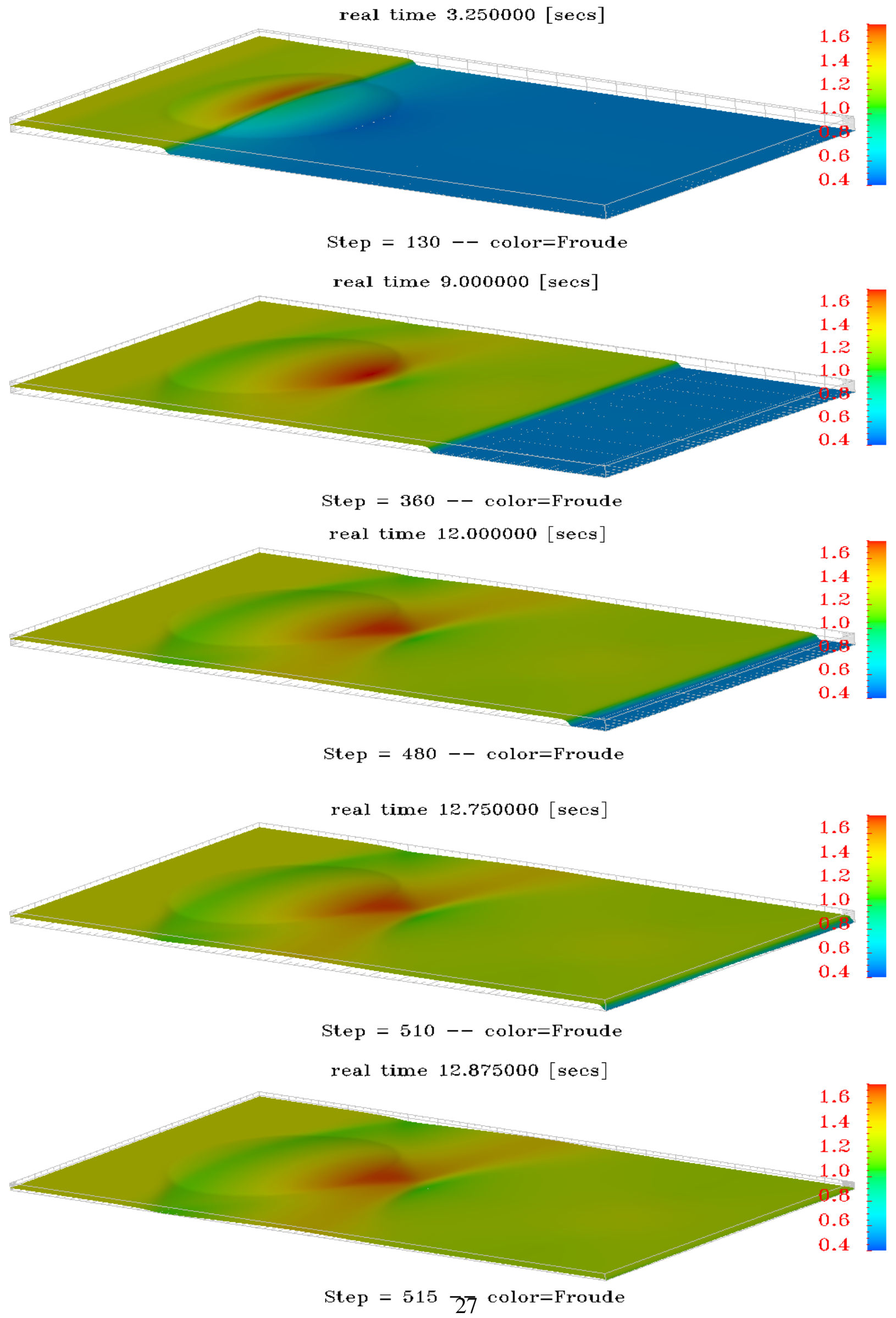

Fig. 13. Froude number: subcritical to supercritical absorbent/dynamic boundary condition. 


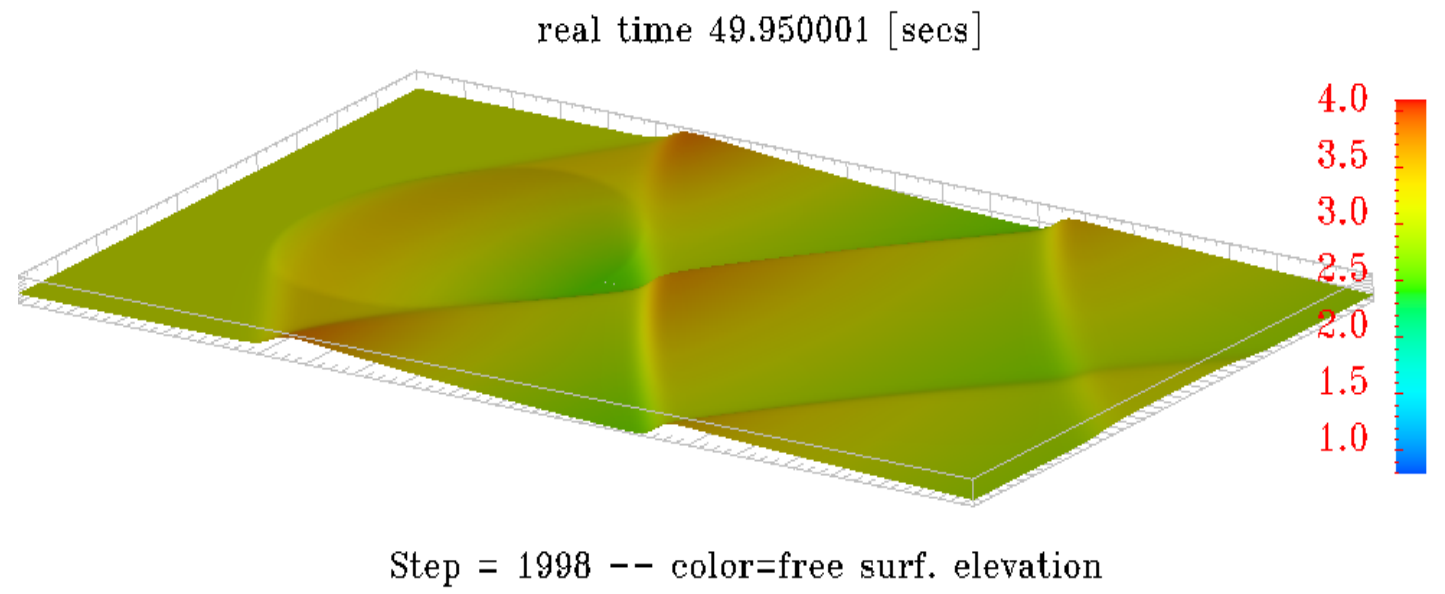

Fig. 14. Converged steady state: free surface elevation

\section{Conclusions}

A general methodology to develop absorbent and dynamic boundary conditions for problems with unknown Riemann invariants is presented. From the computational point of view, the method is only based on computing the advective flux functions and their projected Jacobians onto the normals of the artificial boundaries and then, imposing non-linear constraints via Lagrange multipliers or penalty methods as seen in sections $\S 3.7 .1$ and $\S 3.7 .2$.

Several interesting aspects of ULSAR conditions are put in relevance not only theoretically but also numerically, using a number of tests for different hyperbolic non-linear systems of equations.

Typical problems arising in hydraulic/hydrology areas and how boundary conditions would be imposed are considered.

It is shown that absorbing boundary conditions reduce computational cost by allowing to put the artificial exterior boundary closer to the region of interest.

The performance and transparency of ULSAR conditions are compared with nonreflecting conditions based on Riemann invariants in problems with a closed mathematical form for RI giving similar results in the linear and non-linear region.

It is noticed that the dynamic conditions proposed in this paper are extremely useful when dealing with flow with regime change.

\section{Acknowledgements}

This work has received financial support from Consejo Nacional de Investigaciones Científicas y Técnicas (CONICET, Argentina, PIP 5271/05), Universidad Nacional del Litoral (UNL, Argentina, grants CAI+D 2005-10-64) and Agencia Nacional de Promoción Científica y Tecnológica (ANPCyT, Argentina, grants PICT PME 


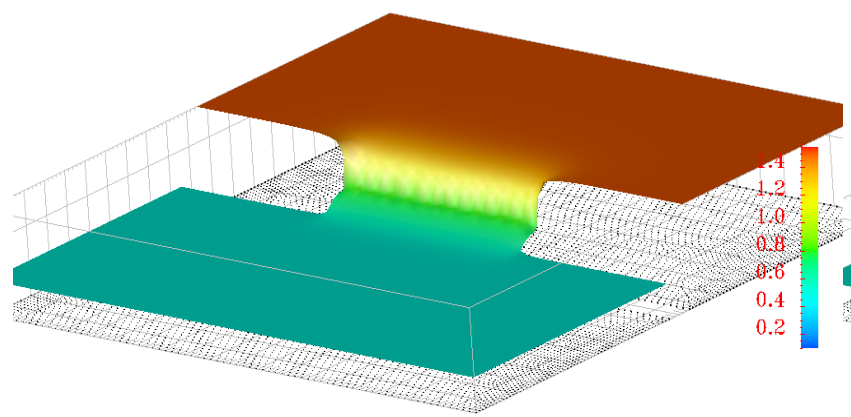

Step $=0--$ color=free surf. elevation

real time 2.100000 [secs]

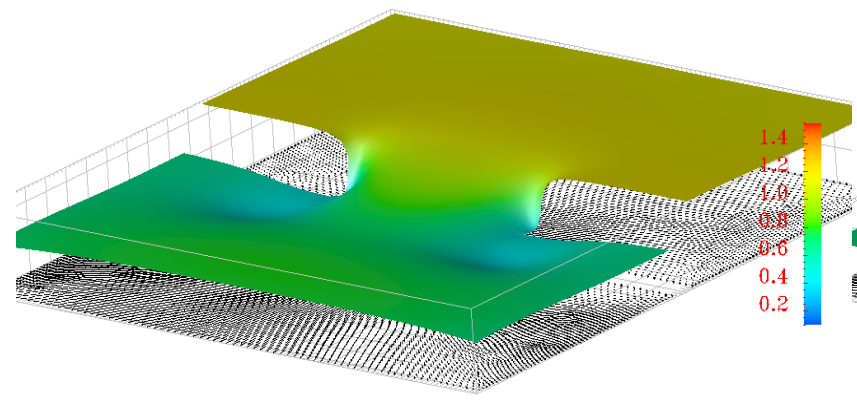

Step $=20--$ color $=$ free surf. elevation
Step $=30--$ color=free surf. elevation

real time 3.100000 [secs]

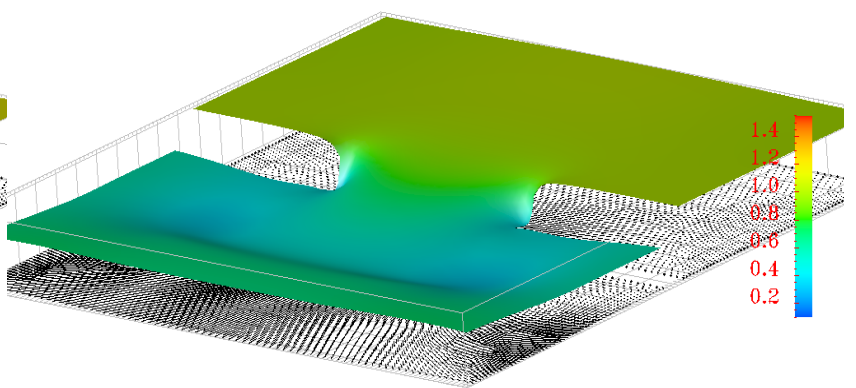

real time 30.100000 [secs]

Fig. 15. Dam break with ULSAR B.C. 

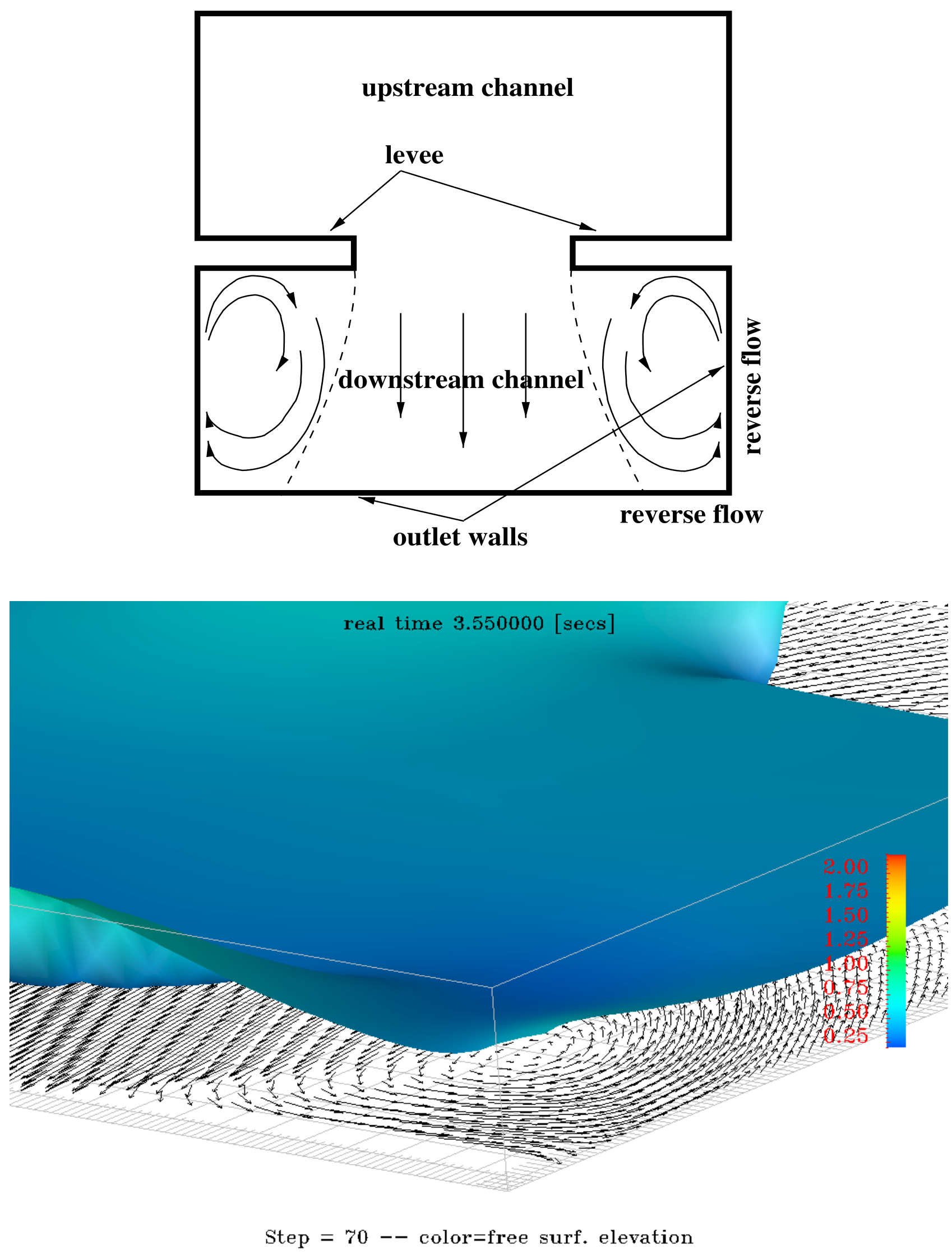

Fig. 16. Reversed flow at the outflow boundary. 
209/2003, PICT-1506/2006, PICTO-23295/2004).

\section{References}

[1] C. Baumann, Storti M., and S Idelsohn. Improving the convergence rate of the PetrovGalerkin techniques for the solution of transonic and supersonic flows. International Journal for Numerical Methods in Engineering, 34:543-568, 1992.

[2] R. Bonet Chaple, N. Nigro, M. Storti, and S. Idelsohn. A discrete non-local (DNL) outgoing boundary condition for diffraction of surface waves. Communications in Numerical Methods in Engineering, 14:849-861, 1998.

[3] J. Broeze and J.E. Romate. Absorbing boundary conditions for free surface wave simulations with a panel method. Journal of Computational Physics, 99:146, 1992.

[4] D. Givoli and J.B.; Keller. A finite element method for large domains. Computer Methods in Applied Mechanics and Engineering, 76:41-66, 1989.

[5] D. Givoli and J.B. Keller. Non-reflecting boundary conditions for elastic waves. Wave Motion, 12:261-279, 1990.

[6] T Hagstrom. Boundary conditions at outflow for a problem with transport and diffusion. Journal of Computational Physics, 69:69-80, 1987.

[7] I. Harari and T.J.R. Hughes. Galerkin least-squares finite element methods for the reduced wave equation with non-reflecting boundary conditions in unbounded domains. Computer Methods in Applied Mechanics and Engineering, 98:411-454, 1992.

[8] T.J.R. Hughes and T.E. Tezduyar. Finite element methods for first-order hyperbolic systems with particular emphasis on the compressible euler equations. Computer Methods in Applied Mechanics and Engineering, 45:217-284, 1984.

[9] J. Nycander, A. McC Hogg, and L.M. Frankcombe. Open boundary conditions for nonlinear channel flow. Ocean Modelling, 24:108-121, 2008.

[10] B.F. Sanders. High-resolution and non-oscillatory solution of the st. venant equations in non-rectangular and non-prismatic channels. Journal of Hydraulic Research, 39(3):321-330, 2001.

[11] M. Storti, J. D’Elía, and S. Idelsohn. Algebraic discrete non-local (dnl) absorbing boundary condition for the ship wave resistance problem. Journal of Computational Physics, 146:570-602, 1997.

[12] M.A Storti, N.M. Nigro, R.R. Paz, and L.D. Dalcin. Dynamic boundary conditions in computational fluid dynamics. Computer Methods in Applied Mechanics and Engineering, 2008. in press.

[13] T.E. Tezduyar. Encyclopedia of Computational Mechanics, volume 3, chapter Finite Element Methods for Fluid Dynamics with Moving Boundaries and Interfaces. John Wiley and Sons, 2004. 
[14] T.E. Tezduyar and T.J.R. Hughes. Finite element formulations for convection dominated flows with particular emphasis on the compressible euler equations. In AIAA Paper 83-0125, Proceedings of AIAA 21st Aerospace Sciences Meeting, Reno, Nevada, 1983.

[15] S.V. Tsynkov. Numerical solution of problems on unbounded domains. a review. Applied Numerical Mathematics, 27:465-532, 1998. 